\title{
Geological and geochemical implications of the genesis of the Qolqoleh orogenic gold mineralisation, Kurdistan Province (Iran)
}

\author{
Batoul Taghipour ${ }^{1}$, Farhad Ahmadnejad ${ }^{2}$ \\ ${ }^{1}$ Department of Earth Sciences, Faculty of Sciences, Shiraz University, Shiraz, Iran \\ e-mail: Taghipour@shirazu.ac.ir, \\ ${ }^{2}$ Department of Earth Sciences, Faculty of Sciences, Shiraz University, Shiraz, Iran \\ *Corresponding author, e-mail: Farhad_sekerter@yahoo.com
}

\begin{abstract}
The Qolqoleh gold deposit is located in the northwestern part of the Sanandaj-Sirjan Zone (SSZ), within the NE-SW trending Qolqoleh shear zone. Oligocene granitoids, Cretaceous meta-limestones, schists and metavolcanics are the main lithological units. Chondrite-normalised REE patterns of the ore-hosting metavolcanics indicate REE enrichment relative to hanging wall (chlorite-sericite schist) and footwall (meta-limestone) rocks. The pattern also reflects an enrichment in LREE relative to HREE. It seems that the LREE enrichment is related to the circulation of $\mathrm{SO}_{4}{ }^{2-}$ and $\mathrm{CO}_{2}$-bearing fluids and regional metamorphism in the Qolqoleh shear zone. Both positive and negative Eu anomalies are observed in shear-zone metavolcanics. These anomalies are related to the degree of plagioclase alteration during gold mineralisation and hydrothermal alteration. In progressing from a metavolcanic protomylonite to an ultramylonite, significant changes occurred in the major/trace element and REE concentration. Utilising an Al-Fe-Ti isocon for the ore-hosting metavolcanics shows that Sc, Y, K, U, P, and M-HREE (except Eu) are relatively unchanged; S, As, Ag, Au, Ca, LOI, $\mathrm{Rb}$ and LREE are enriched, and $\mathrm{Sr}, \mathrm{Ba}, \mathrm{Eu}, \mathrm{Cr}, \mathrm{Co}$ and Ni decrease with an increasing degree of deformation. Based on geochemical features and comparison with other well-known shear zones in the world, the study area is best classified as an Isovolume-Gain (IVG) type shear zone and orogenic type gold mineralisation.

Based on the number of phases observed at room temperature and their microthermometric behaviour, three fluid inclusion types have been recognised in quartz-sulphide and quartz-calcite veins: Type I monophase aqueous inclusions, Type II two-phase liquid-vapour (L-V) inclusions which are subdivided into two groups based on the homogenisation temperature $\left(\mathrm{T}_{\mathrm{h}}\right)$ : a) L-V inclusions with $\mathrm{T}_{\mathrm{h}}$ from 205 to $255^{\circ} \mathrm{C}$ and melting temperature of last ice $\left(\mathrm{T}_{\mathrm{m}}\right)$ from -3 to $-9^{\circ} \mathrm{C}$. b) L-V inclusions with higher $\mathrm{T}_{h}$ from 335 to $385^{\circ} \mathrm{C}$ and $\mathrm{T}_{\mathrm{m}}$ from -11 to $-16^{\circ} \mathrm{C}$. Type III three-phase carbonic-liquid inclusions (liquid water-liquid $\mathrm{CO}_{2}$-vapour $\mathrm{CO}_{2}$ ) with $\mathrm{T}_{\mathrm{h}}$ of $345-385^{\circ} \mathrm{C}$. The mean values of the density of ore-forming fluids, pressure and depth of mineralisation have been calculated to be $0.79-0.96 \mathrm{gr} / \mathrm{cm}^{3}, 2 \mathrm{kbar}$ and $7 \mathrm{~km}$, respectively. The $\delta^{18} \mathrm{O}_{\text {water }}$ and $\delta D$ values of the gold-bearing quartz-sulphide veins vary from $7.2 \%$ o to $8 \%$ o and $-40.24 \%$ o to $-35.28 \%$, respectively, which are indicative of an isotopically heavy crustal fluid and likely little involvement of meteoric fluid. The $\delta^{18} \mathrm{O}_{\text {water }}$ values of the quartz-calcite veins have a range of $-5.31 \%$ o to $-3.35 \%$, and the $\delta \mathrm{D}$ values of $-95.65 \%$ o to $-75.31 \%$, which are clearly lower than those of early-stage quartz-sulphide-gold veins, and are close to the meteoric water line. Based on comparisons of the D-O isotopic systematics, the Qolqoleh ore-mineralising fluids originated from metamorphic devolatilisation of Cretaceous volcano-sedimentary piles. Devolatilisation of these units occurred either synchronously with, or postdates, the development of penetrative (ductile) structures such as shear zones and during overprinting brittle deformation.
\end{abstract}

Keywords: fluid inclusion, $\delta^{18} \mathrm{O}$ and $\delta \mathrm{D}$ isotopes, rare earth and trace element mobility, Sanandaj-Sirjan Zone, Iran 


\section{Introduction}

Orogenic gold deposits are related to collision settings and active orogenic belts (Bierlein et al., 2001). They formed during compressional to transpressional deformation processes at convergent plate margins in accretionary and collisional orogens (Groves et al., 1998, 2000; Goldfarb et al., 2001). These deposits are epigenetic and structurally controlled, and based on their depth of formation, are divided into epizonal, mesozonal, and hypozonal subtypes (Groves et al., 1998, 2000; Goldfarb et al., 2005).

Except for the Zartorosht and Muteh gold deposits, which are located in the southeastern and central parts of the Sanandaj-Sirjan Zone (SSZ) (Fig. 1), respectively, no major orogenic gold deposits have been recognised in Iran. Nevertheless, several gold occurrences have recently been explored in the northwestern SSZ (Fig. 2). Previous studies of the Qolqoleh gold deposit have outlined some aspects of gold mineralisation (Aliyari et al., 2009) and the behaviour of major, trace and REEs during hydrothermal alterations (Aliyari et al., 2012).

Many researchers have investigated shear-zone formation and various aspects of elements mobility and volume/mass changes during mylonitisation (Sinha et al., 1986; O'Hara, 1988; Dipple et al., 1990; Selverstone el al., 1991; Goddard \& Evans, 1995; Condie \& Sinha, 1996; Hippertt, 1998; Kwon et al., 2009). Some investigations suggest that almost all major and many trace elements can be mobilised during mylonitisation (e.g., Dickin, 1988; O'Hara \& Blackburn, 1989; Selverstone el al., 1991; Glazner \& Bartley, 1991; Condie \& Sinha, 1996; Rolland et al., 2003). REE geochemistry is often used to characterise protoliths of metamorphosed and highly altered rocks (e.g., Taylor \& McLennan, 1985; Vance \& Condie, 1987; Murray et al., 1990). However, these elements can also be mobilised during deformation and metamorphism (Dickin, 1988; O'Hara, 1990; Goddard \& Evans, 1995; Condie \& Sinha, 1996).

The behaviour of REE and $\mathrm{Y}$ is strongly decoupled from that of other elements (except U-Ta-Th-

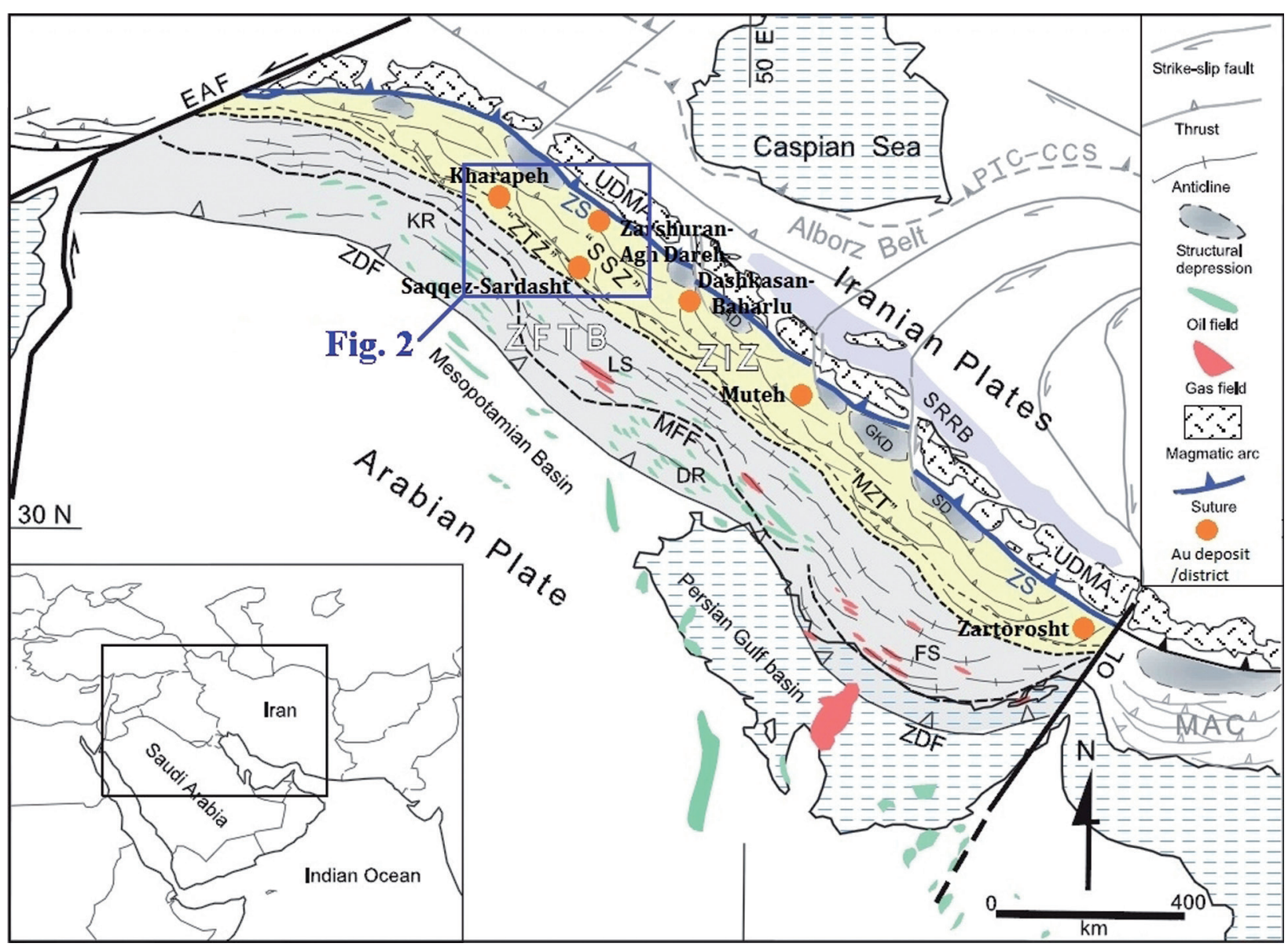

Fig. 1. Tectonic subdivision zones of the Zagros orogen, western Iran, and location of known gold deposits in the Sanandaj-Sirjan Zone (modified after Berberian, 1976; Eftekhar-Nezhad, 1981; Niromand et al., 2011). 
Hf) in the shear zone (Rolland et al., 2003). Regarding REE mobility in shear zones, it is necessary to consider the mineralogical equilibria between fluids and REE-bearing minerals, as well as effects of $\mathrm{pH}$, temperature, redox conditions and of potential ligands present in the mineralising fluid (Bau, 1991; Fourcade et al., 1989; Rolland et al., 2003). Precipitation and dissolution of REE-bearing minerals at various stages of shear zone development is an in- dicator of changes in fluid chemistry in the individual shear zone.

To study element behaviour during the deformation progress in shear zones, element distribution can be evaluated with Al-Ti-Fe isocon plots (Condie \& Sinha, 1996). Based on the isocon method and volume or mass change during deformation, shear zones are classified into three groups: (1) volume-conservative (isovolume); (2) volume-loss,

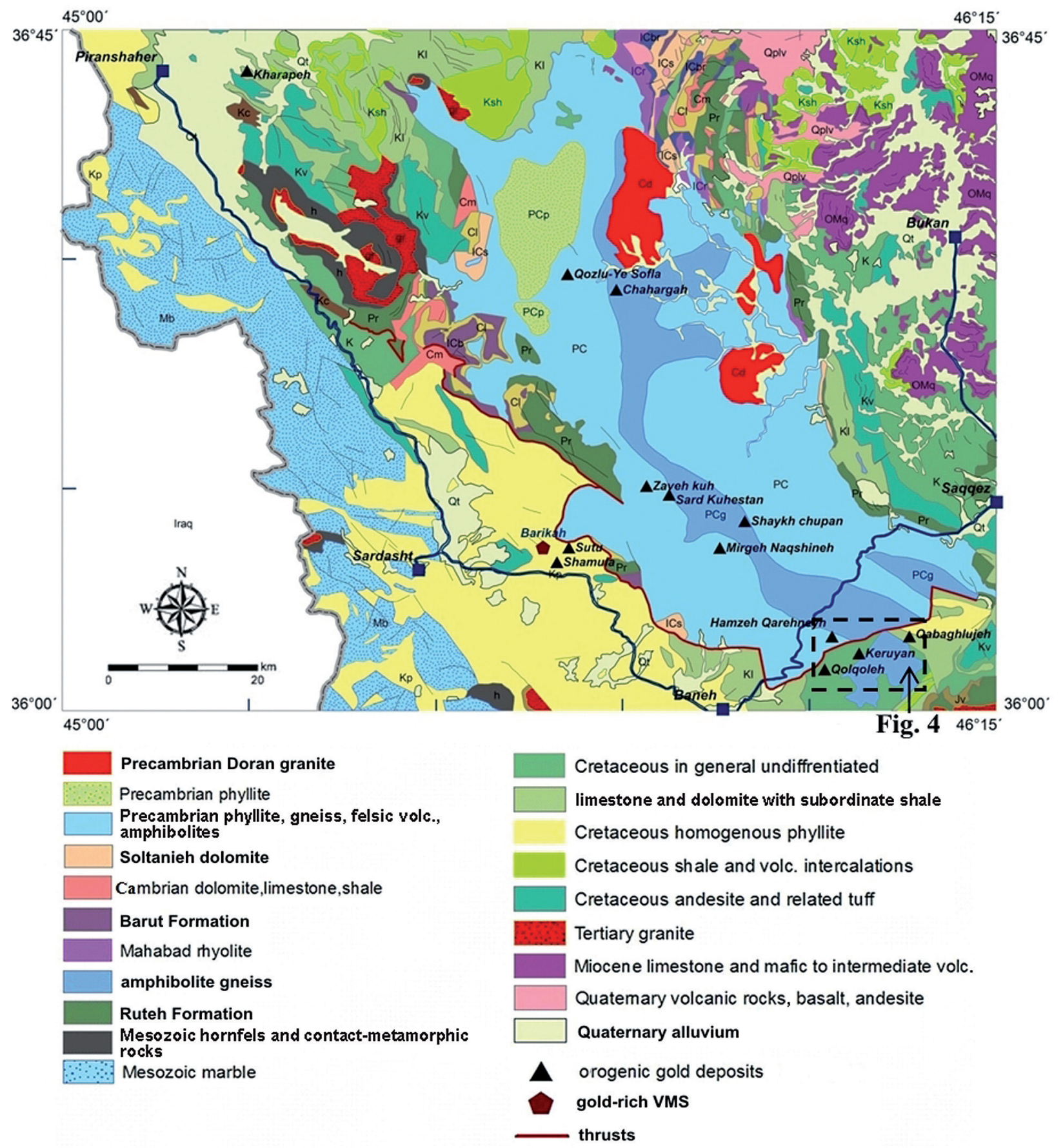

Fig. 2. Simplified geological map of the Saqqez-Sardasht-Piranshaher area (after Eftekhar-Nezhad, 1973) showing gold occurrences. 
and (3) volume-gain (Condie \& Sinha, 1996; Kwon et al., 2009).

This paper focuses on the geochemistry of trace elements and REE during transition from protomylonite to ultramylonite conditions in the Qolqoleh shear zone. The best-fit Al-Ti-Fe isocon method is used to determine chemical and volume change during mylonitisation and development of shear zone. Detailed fluid inclusion analyses and stable isotope data are used to determine possible sources of mineralising fluid, and to propose a model for the Qolqoleh shear zone evolution.

\section{Methodology}

A total of about ninety-five surface rock samples were collected for petrographical and geochemical investigations. Twenty fine microscopic thin sections were prepared for petrographical studies. Nine intrusive rocks were analysed for major and trace elements using X-ray fluorescence (XRF) (Philips PW2400, equipped with an Rh-tube) at the Kansaran Binalood laboratory, Pardis Science and Technology Park, Iran. The detection limit for the major oxides is about $0.01 \mathrm{wt} \%$, but it varies for trace elements (in ppm): $\mathrm{Nb}$ (1); Th and $\mathrm{Rb}$ (2); $\mathrm{Y}$ and $\mathrm{Ni}$ (3); Cr, V and $\mathrm{Zr}$ (4); $\mathrm{Sr}$ (5); and $\mathrm{Ba}(10)$. In addition, 18 metavolcanics, chlorite-sericite schist (hanging wall) and meta-limestone (footwall) samples were analysed for trace and rare earth elements using inductively coupled plasma mass spectrometry (ICP-MS) in the ACME Analytical Laboratories Ltd., Vancouver, Canada. Microthermometric studies were carried out to investigate the nature and evolution of the mineralising fluids, and to determine physicochemical parameters of gold deposition with respect to the regional metamorphic and magmatic conditions. The fluid inclusion studies were carried out on 10 polished, thin-sectioned quartz specimens. These studies were carried on both concordant gold-bearing quartz-sulphide, and discordant quartz-calcite veins and veinlets. Microthermometric measurements were obtained on a Linkhan THM 600 heating/freezing stage with a temperature range of -196 to $600^{\circ} \mathrm{C}$, mounted on a Zeiss-Olympus petrographic microscope at the Iran Mineral Processing Research Center and Esfahan University. The system was calibrated with synthetic fluid inclusions (Sterner \& Bodnar, 1984) at temperatures $-56.6^{\circ} \mathrm{C}, 0.0^{\circ} \mathrm{C}$, and $374.1^{\circ} \mathrm{C}$. To avoid decrepitation of the inclusions, freezing was carried out prior to heating. For all final melting temperatures of $\mathrm{CO}_{2}$, precision is $\pm 0.1^{\circ} \mathrm{C}$. In this study, about 145 fluid inclusions were investigated by microthermometry. To minimise effects of post-entrapment modifications such as necking down and partial or total leakage, inclusions that have been affected by these phenomena, and those in very highly-deformed quartz grains and secondary inclusions were avoided. Ten quartz vein samples (concordant gold-bearing quartz-sulphide veins and discordant quartz-calcite veins) were selected for microthermometric measurements. The nature of inclusions, their contents (liquid-solid-gas), distribution patterns, types (primary, secondary, pseudosecondary), post-entrapment modification, shape, size and phase categories were studied and documented using a petrographical microscope at varying magnifications following the techniques outlined by Roedder (1984) and Touret (2001).

Four quartz samples were analysed for $\delta^{18} \mathrm{O}$ and $\delta \mathrm{D}$. The oxygen isotopic composition of two types of quartz was measured on $\mathrm{O}_{2}$ evolved from laser-assisted fluorination and deuterium composition of inclusion fluids in both quartz vein types was measured on $\mathrm{H}_{2}$. Handpicked sampling was carried out from both concordant gold-bearing quartz-sulphide veins and discordant quartz-calcite veins. Quartz from these veins was crushed into $0.1-0.5 \mathrm{~mm}$ grains. All isotope analyses were performed in the Cornell stable isotope laboratory at the University of Cornell, USA. The external precision and accuracy for both $\delta^{18} \mathrm{O}$ and $\delta \mathrm{D}$ is $\pm 0.2 \%$. Results for $\delta^{18} \mathrm{O}$ and $\delta \mathrm{D}$ are compared against the Vienna Standard Mean Ocean Water (V-SMOW).

\section{Geological setting}

During the Middle to Late Triassic, the Neo-Tethyan Ocean had opened up between Iran and Arabia, with two different platforms on either side (Berberian and King, 1981). The subduction of Neo-Tethyan oceanic lithosphere beneath the southwestern border of central Iran eventually led to suturing of the Iranian and Arabian continents (e.g., Berberian and King, 1981; Alavi, 1994), and subsequent continental convergence formed the Zagros Orogenic Belt (Fig. 1).

The Zagros orogen is part of the extensive Alpine-Himalayan orogenic belt that formed during collision of the northeastern margin of the Arabian continental plate with the central Iranian microcontinent in the Cretaceous-Cenozoic (Berberian \& King, 1981; Alavi, 1994; Mohajjel et al., 2003). The northwestern boundary of the Zagros orogen is chosen to be the East Anatolian strike-slip fault in southeastern Turkey, and the southeastern boundary of the Zagros orogen is the Oman Line (Alavi, 2007). This orogen consists of three distinctive par- 
allel tectonic zones from the northeast to southwest including (Fig. 1) 1) the Cenozoic Urumieh-Dokhtar magmatic (UDMA); 2) the Sanandaj-Sirjan Zone (SSZ), and 3) the Zagros fold belt (Alavi 1994, 2007; Mohajjel et al. 2003; Agard et al. 2005; Sheikholeslami et al. 2008; Niromand et al., 2011).

The Sanandaj-Sirjan Zone, which hosts Qolqoleh and other gold occurrences, covers a region of polyphase deformation, the latest reflecting the collision of the Arabian continent and the central Iranian microcontinent, and subsequent southward propagation of the fold-thrust belt (Alavi, 1994; Mohajjel et al., 2003, Sarkarinejad and Alizadeh, 2009). It is a major NW-SE trending structural zone that extends for more than 1,500 km length and ap- proximately $200 \mathrm{~km}$ width to the northeast of the Zagros Thrust System and contains the metamorphic core of the Zagros continental collision zone in western Iran (Baharifar et al., 2004; Mohajjel et al., 2003; Sarkarinejad and Alizadeh, 2009). Different hypotheses have been proposed for the mechanism and exact timing of subduction and collision along this active margin (i.e., the Zagros orogen). These hypotheses can be classified into two groups according to the timing of initial subduction and continental collision (Fig. 3): 1) Many researchers believe that the initiation of oceanic subduction occurred in the Early to Late Cretaceous (Berberian \& Berberian, 1981; Moinevaziri, 1985; Alavi, 1994) and continental collision occurred in the Late Cre-

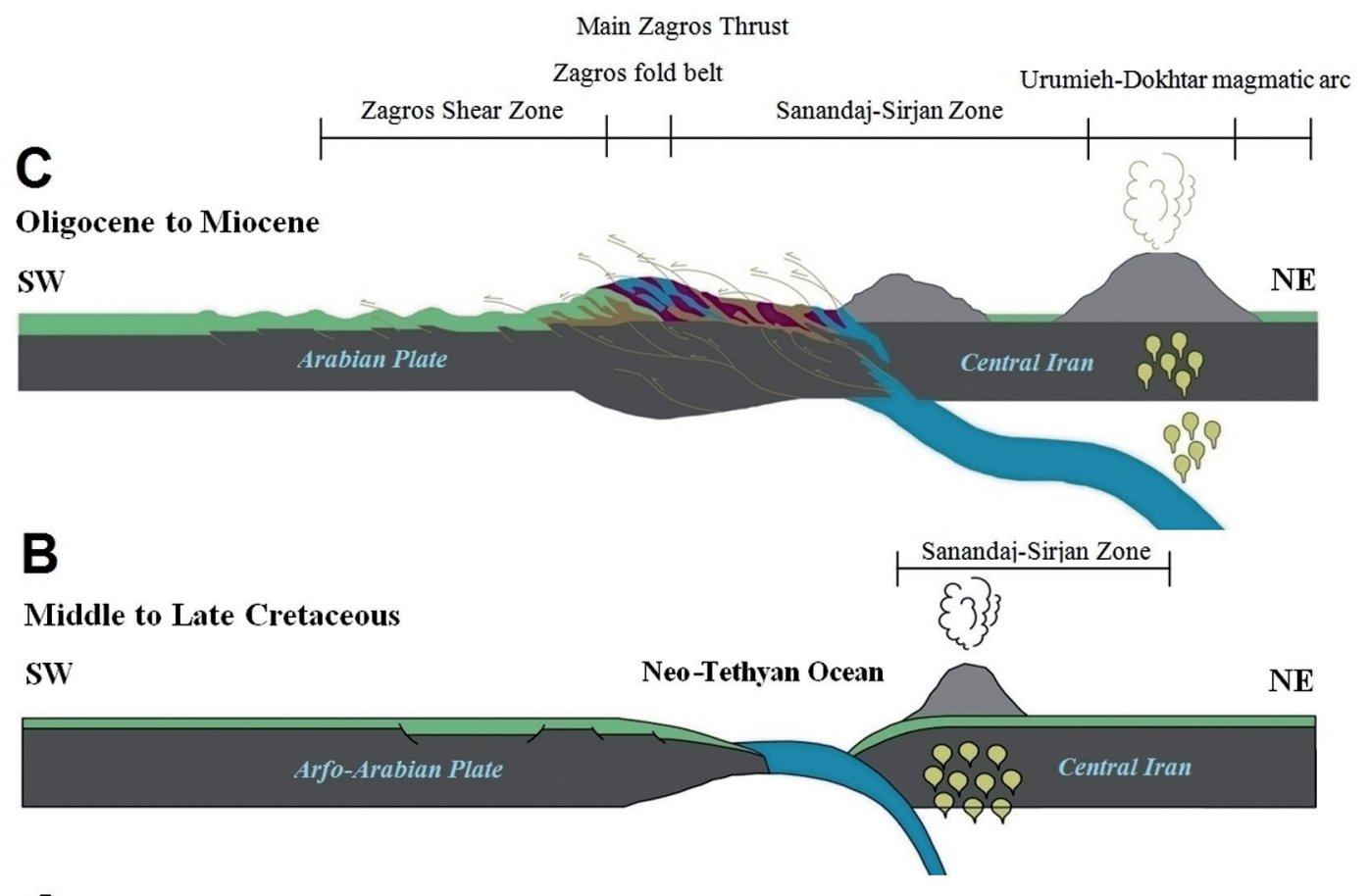

A

Permian to Triassic

SW

Neo-Tethyan Ocean

NE

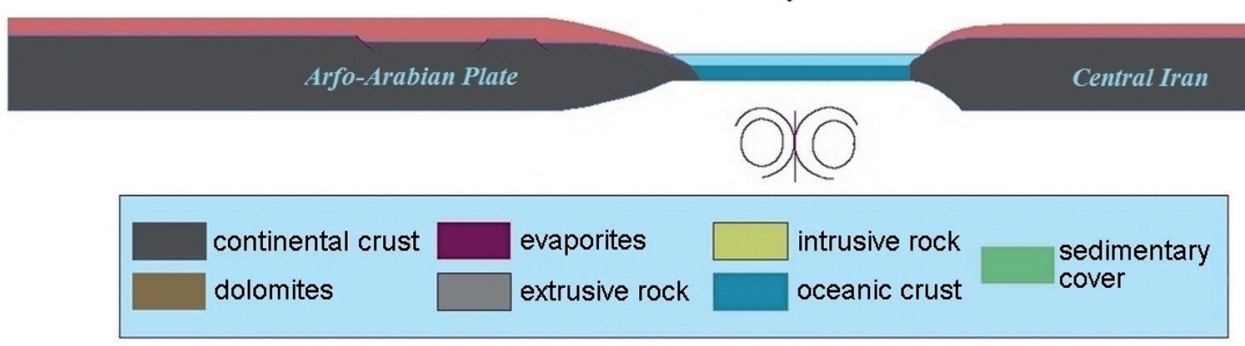

Fig. 3. Geotectonic model for the evolution of the northern part of the Sanandaj-Sirajan Zone (modified after Alavi, 1994; 2004; Agard et al., 2005; Niroomand et al., 2011).

A - Neo-Tethys ocean opening in the Permian. The gold occurrences are formed in the western margin of central Iran; B - Subduction of Neo-Tethys oceanic crust started in the early Mesozoic, and the main part was subducted under central Iran by the end of the Cretaceous. Arc-related subduction was developed in the northern part of the Sanandaj-Sirjan zone; C - Final collision of the Arabian plate and central Iran in the Oligocene-Miocene (late Cenozoic). 
taceous-Early Paleocene (Alavi, 1994, 2004), and 2) In contrast, some workers consider the timing of initial subduction to be Late Triassic-Early Jurassic (Agard et al., 2005; Arvin et al., 2007; Omrani et al., 2008) and suggest that the continental collision occurred in the Neogene (Berberian, 1983; Shahabpour, 2005). Deformed and mainly Mesozoic metasedimentary and metavolcanic rocks are located in the northwest to southeast of Iran (Mohajjel and Fergusson 2000) (Fig. 2). In addition to Jurassic-Eocene subduction-related intrusions in SSZ along the northeastern margin (e.g., the Urumieh-Dokhtar volcanic arc), there is a belt of Cretaceous flysch and volcanic rocks (Figs. 1, 2) in the northwestern part of the SSZ (Azizi and Moinevaziri 2009). Palaeozoic and Mesozoic rocks of the SSZ are metamorphosed and highly deformed in the Zagros orogenic belt (Alavi, 1994; Berberian, 1995). Eftekhar-Nezhad (1981) and Ghasemi \& Talbot (2006) subdivided SSZ into two parts: 1) A belt of phyllite, metavolcanics and intrusive felsic rocks in the northwestern part of the SSZ, which were affected by deformation in the Late Cretaceous, and 2) Pre-Jurassic metamorphics and highly deformed post-Triassic sediments in the southeastern part. Recent studies by Azizi \& Moinevaziri (2009) indicate that Cretaceous volcanic rocks are developed as a separate belt in the northern part of the SSZ between Nahavand and
Urumieh. This belt measures $15-20 \mathrm{~km}$ in width and $200-300 \mathrm{~km}$ in length, and extends in a NWSE direction parallel to the main Zagros fault zone (Mohajjel et al., 2003; Azizi \& Moinevaziri, 2009). According to Niromand et al. (2011), the Cretaceous metamorphosed volcano-sedimentary rocks of the Sardasht-Piranshahr-Saqqez zone host the gold deposits of the northwestern SSZ. These units underwent greenschist facies metamorphism (Fig. 2).

\subsection{Geology of the Qolqoleh gold deposit}

The Qolqoleh gold deposit is located at $36^{\circ} 08^{\prime} 08^{\prime \prime}$ $\mathrm{N}, 46^{\circ} 06^{\prime} 08^{\prime \prime} \mathrm{E}$ in the northwestern part of the SSZ, $50 \mathrm{~km}$ southwest of Saqqez (Figs. 1, 2). This deposit is one of the gold occurrences of the Saqqez-Sardasht-Piranshaher gold-bearing zone. This zone has undergone deformation and greenschist facies metamorphism. Geochemical studies indicate that gold mineralisation in this zone is related to the ductile and brittle shear zones, which are suitable pathways for transportation and deposition of metals from ore-bearing fluids (Aliyari et al., 2007). Rock units exposed in the study area include granitoid intrusive (as widespread outcrops of gneiss and granite-gneiss), phyllite, meta-limestone, chlorite-sericite schist, and metavolcanics (Fig. 4). Meta-

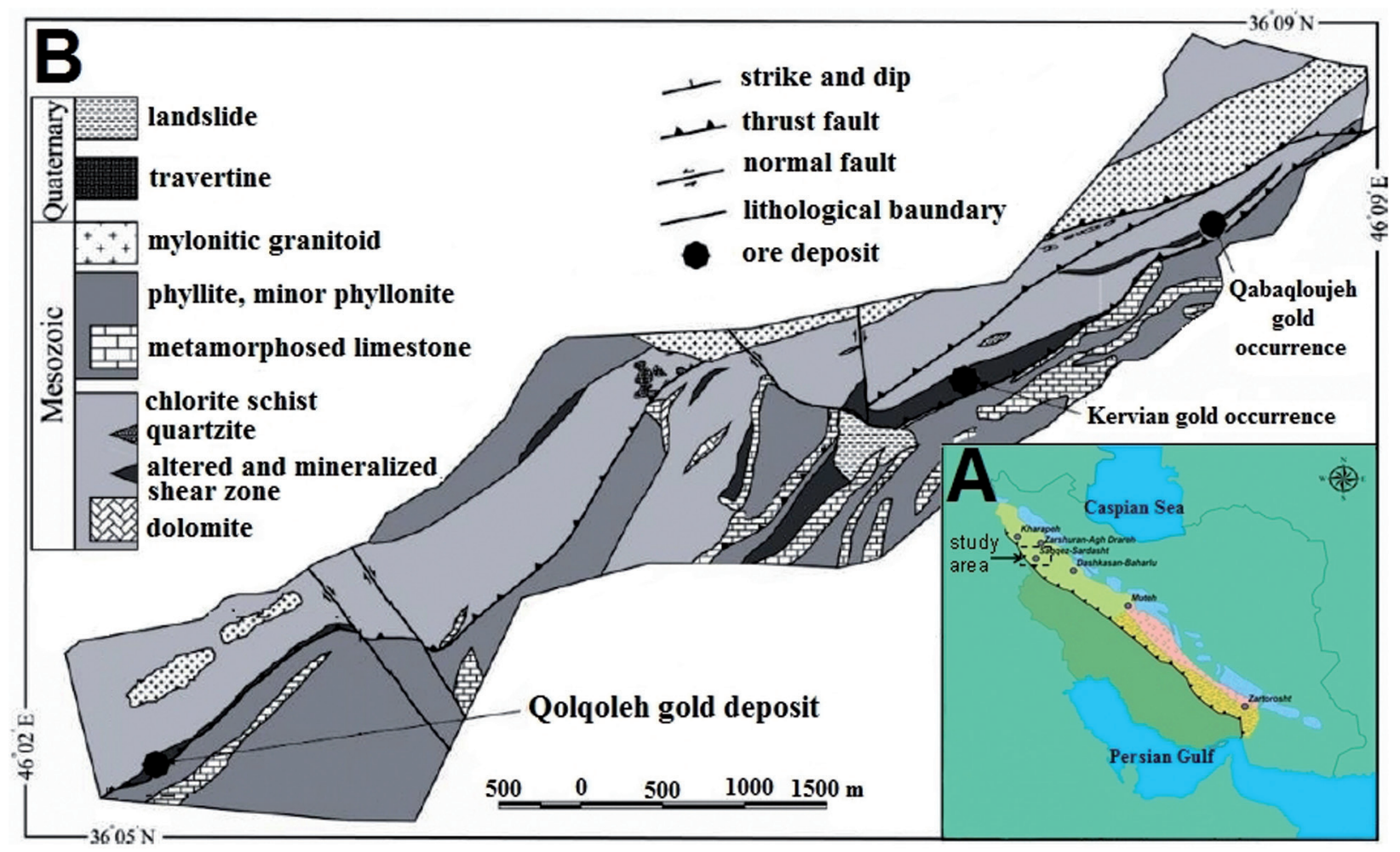

Fig. 4. A - Location of the study area within the Sanandaj-Sirjan metamorphic zone; B - Geological map of the study area showing location of major ore bodies and faults (modified after Mohajjel, 2000). 
morphics, including chlorite-sericite schists, are the main component of hanging wall, whereas phyllite and meta-limestone constitute footwall. The area is affected by a NE-SW trending ductile-brittle shear zone. All rock units show different deformation intensity from less deformed to highly deformed. Propylitic, phyllic, and silicic are the main alteration zones in the area. Gold mineralisation is related to highly deformed silicified mylonite and ultramylonite units (Aliyari et al., 2009). The main mineralised zone is lens shaped, measuring about $2 \mathrm{~km}$ in length and $250 \mathrm{~m}$ in width.

Based on geometry, texture, and mineralogy, the quartz veins are classified into concordant and discordant types. The discordant quartz veins, as saddle shapes and/or as fracture fillings, were developed along extensional fractures in the fold limb propylitic, phyllic, and silicic are the main alteration zones. The concordant quartz veins host gold mineralisation, although some gold concentrations are present in discordant veins in the adjacent sulphidised altered wall rock.

There are three deformational stages $\left(\mathrm{D}_{1}, \mathrm{D}_{2}\right.$ and $\mathrm{D}_{3}$ ) in the structural history of the Sananda-Sirjan zone (Mohajjel et al., 2003). Mesoscopic fold and planar schistosity took place during $\mathrm{D}_{1}$ deformation during the Late Jurassic-Early Cretaceous. $\mathrm{D}_{2}$ deformation produced the shear zone trending NW-SE due to Afro-Arabian collision with the southwestern part of SSZ. The $\mathrm{D}_{2}$ deformation episode led to the formation of dominant structures in the Qolqoleh (Mohajjel, 1997; Aliyari et al., 2009). Brittle to ductile shear zones formed as the $\mathrm{D}_{3}$ deformation type in the Early Cenozoic.

\section{Vein mineralogy}

Field investigation shows two types of quartz veins: quartz-carbonate and quartz-sulphide.

\subsection{Quartz-carbonate veins}

These veins are characterised by low sulphide mineralisation. Mineralogy includes quartz (60 $\mathrm{wt} \%)$, calcite $(15 \mathrm{wt} \%)$, sericite-biotite $(10 \mathrm{wt} \%)$, plagioclase (5 wt \%), pyrite $(<5 \mathrm{wt} \%)$, and chlorite and chalcopyrite (1-3 wt \%). Quartz-carbonate veins cropped out mainly as dilation veins (Fig. 5A). The cross-cutting relationship suggests that silicification postdated carbonatisation (Fig. 5B). Relict patches of carbonates and rounded margins are common in the quartz matrix (e.g. Fig. 6A, B).

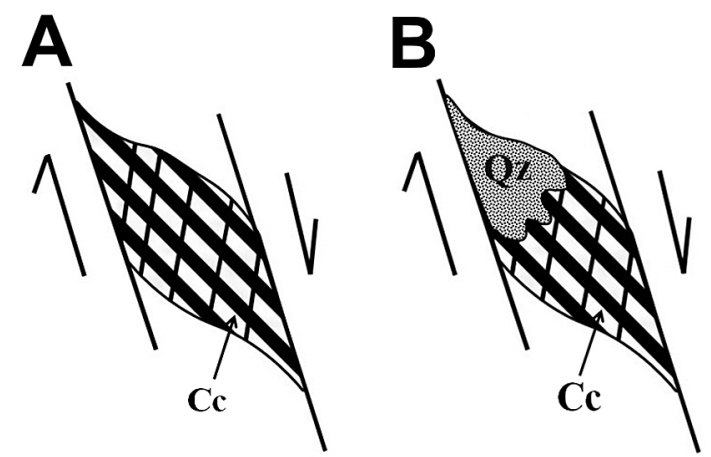

Fig. 5. Schematic sketch presenting the formation of a quartz-carbonate dilation vein.

A - Vein dilation and calcite replacement; B - Quartz replacement with some mineralisation.

\subsection{Quartz-sulphide veins}

Quartz-sulphide veins extend along mylonite induced foliations and host the greatest sulphide mineralisation in the area. These NW-SE trending veins are mostly widespread and occur in the mineralised parts of shear zones (Fig. 6C). The mineralogy includes quartz (70 wt \%), plagioclase (10 wt \%), biotite-sericite $(10 \mathrm{wt} \%)$, and pyrite (> $5 \mathrm{wt} \%)$; chlorite and chalcopyrite are present as accessory minerals (Fig. 6D).

\section{Geochemistry}

Major and trace element geochemistry of nine samples from plutonic rocks (Table 1) suggests that the Qolqoleh gold deposit resulted from a sub-alkaline magma emplacement in a volcanic arc setting (Fig. 7A, B).

Except for a very high fluid/rock ratio (Michard, 1989), REEs with low solubility are relatively immobile during low-grade metamorphism, weathering and hydrothermal alteration (Rollinson, 1993). Some studies (e.g., Dickin, 1988; O'Hara, 1990; Glazner \& Bartley, 1991) have shown that REE can be remobilised during deformation and metamorphism. According to Lottermoser (1992), very high fluid/rock ratios are necessary to make significant changes in the REE pattern of silicate-rich rocks during diagenesis and metamorphism.

The REE diagrams (Fig. 8) show enrichment in the shear zone $(\Sigma \mathrm{REE}=247 \mathrm{ppm})$ relative to hanging wall and footwall units $(\Sigma \mathrm{REE}=143 \mathrm{ppm}$ and $\Sigma$ REE $=50$ ppm, respectively; Table 2 ). These patterns also reflect enrichment in LREE relative to HREE. In addition, it shows a stronger negative LREE slope compared to HREE. Both positive and 

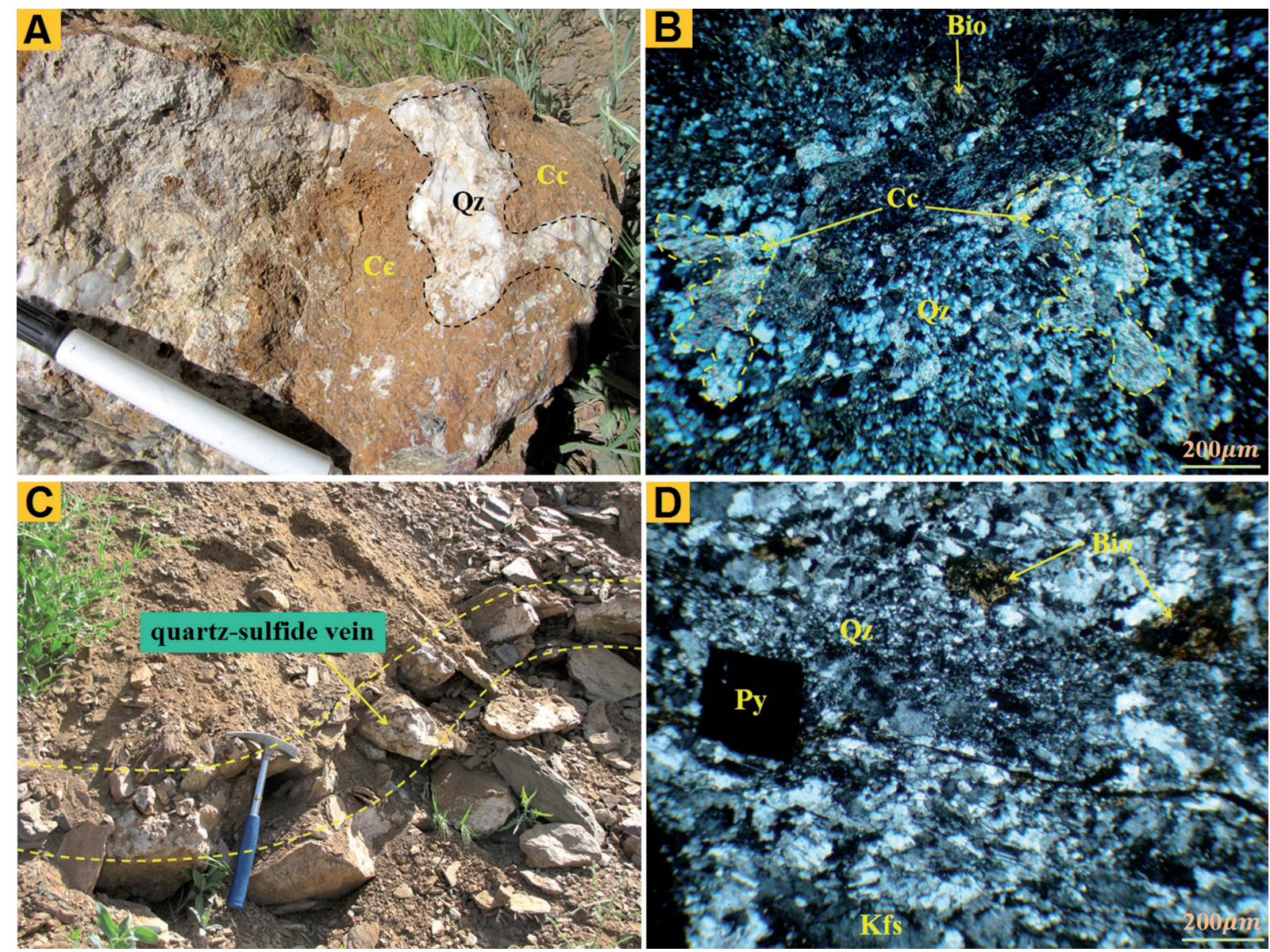

Fig. 6. A, B - Hand specimen and photomicrograph of quartz-carbonate vein; C, D - Quartz-sulphide veins along the Qolqoleh shear zone and photomicrograph of the same.

negative Eu anomalies are observed in the metavolcanics of the shear zone (e.g. Fig. 8A, B). Europium replaces $\mathrm{Ca}$ in plagioclase, so the negative and positive $\mathrm{Eu}$ anomalies correspond to the degree of pla- gioclase decomposition (Kikawada, 2001) or addition of plagioclase during hydrothermal alteration.

Relative depletion of REE and absence of a Eu anomaly in the hanging wall (sericite-chlorite
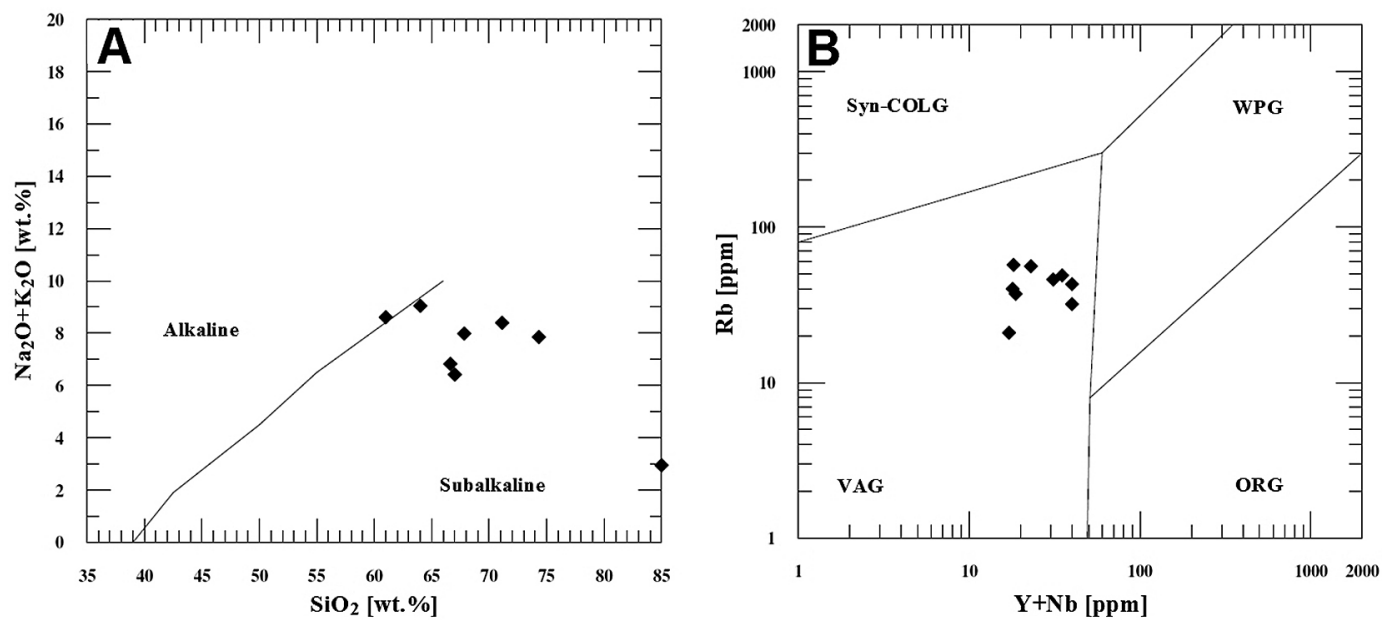

Fig. 7. A - Discrimination diagram of alkaline-subalkaline series and position of samples studied (boundary after Irvine and Baragar, 1971); B - Rb vs ( $\mathrm{Y}+\mathrm{Nb})$ diagram for classification of granitoids and position of the Qolqoleh samples (boundaries after Pearce et al., 1984). 
Table 1. Major and trace element composition of the intrusive body in the Qolqoleh deposit, Iran; $<1$ means below detection limit.

\begin{tabular}{|c|c|c|c|c|c|c|c|c|c|}
\hline Samples & Gh.2.15 & Gh.2.2 & Gh.3.3 & Gh.2.5 & Gh.2.23 & Gh.4.3 & Gh.4.7 & Gh.4.16 & Gh.4.18 \\
\hline Samples & Gnd & Gn & $\mathrm{Gn}$ & Gnd & Dr & Gnd & Gn & Gn & Gn \\
\hline $\mathrm{SiO}_{2}(\%)$ & 67.13 & 85.06 & 85.82 & 64.11 & 61.93 & 66.61 & 74.32 & 71.12 & 67.83 \\
\hline $\mathrm{TiO}_{2}$ & 0.41 & 0.38 & 0.39 & 0.42 & 0.37 & 0.62 & 0.58 & 0.95 & 0.71 \\
\hline $\mathrm{Al}_{2} \mathrm{O}_{3}$ & 13.35 & 8.53 & 7.1 & 14.32 & 17.01 & 17.70 & 14.17 & 14.36 & 16.94 \\
\hline $\mathrm{Fe}_{2} \mathrm{O}_{3}$ & 4.2 & 1.32 & 1.64 & 4.32 & 5.52 & 4.05 & 1.67 & 2.75 & 2.86 \\
\hline $\mathrm{MnO}$ & 0.01 & 0.02 & 0.04 & 0.09 & 0.02 & 0.06 & 0.03 & 0.04 & 0.06 \\
\hline $\mathrm{MgO}$ & 2.2 & 0.5 & 0.32 & 0.57 & 3.24 & 1.4 & 0.17 & 0.43 & 0.54 \\
\hline $\mathrm{CaO}$ & 4.35 & 0.83 & 0.31 & 3.58 & 0.27 & 0.84 & 0.27 & 0.48 & 0.72 \\
\hline $\mathrm{Na}_{2} \mathrm{O}$ & 3.34 & 1.63 & 2.89 & 4.47 & 4.9 & 3.60 & 3.62 & 4.36 & 4.11 \\
\hline $\mathrm{K}_{2} \mathrm{O}$ & 2.9 & 1.32 & 1.62 & 4.58 & 3.71 & 3.32 & 4.23 & 4.03 & 3.87 \\
\hline $\mathrm{P}_{2} \mathrm{O}_{5}$ & 0.21 & 0.27 & 0.19 & 0.17 & 0.23 & 0.26 & 0.11 & 0.05 & 0.07 \\
\hline LOI & 3.07 & 0.89 & 0.74 & 2.92 & 3.1 & 1.71 & 0.95 & 1.26 & 1.88 \\
\hline Total & 98.96 & 100.28 & 100.67 & 99.31 & 99.90 & 100.07 & 100.12 & 99.83 & 99.54 \\
\hline $\mathrm{Ba}(\mathrm{ppm})$ & 10.5 & 780 & 1018 & 870 & 1020 & 780 & 290 & 506 & 638 \\
\hline $\mathrm{Rb}$ & 37.2 & 20.91 & 40.1 & 57.15 & 56 & 43 & 49 & 32 & 46 \\
\hline $\mathrm{Sr}$ & 860 & 910 & 987 & 740 & 845 & 810 & 760 & 920 & 870 \\
\hline $\mathrm{Zr}$ & 93 & 101 & 110 & 89 & 98 & 169 & 85 & 107 & 98 \\
\hline $\mathrm{Y}$ & 8.71 & 10.1 & 12 & 7.2 & 11.01 & 26 & 24 & 21 & 15 \\
\hline $\mathrm{Nb}$ & 10 & 7 & 6 & 11 & 12 & 14 & 11 & 19 & 16 \\
\hline $\mathrm{Ga}$ & 20 & 21 & 23 & 19 & 20 & 18 & 20 & 29 & 23 \\
\hline $\mathrm{Ag}$ & 1 & 1 & 1 & $<1$ & $<1$ & 1 & 1 & 1 & 1 \\
\hline Sn & 2 & 1 & 3 & 2 & 1 & 2 & 1 & $<1$ & 1 \\
\hline $\mathrm{Ta}$ & 1.1 & 1.6 & 1.5 & 1.7 & 1.4 & 2.1 & 1.4 & 1.1 & 1.7 \\
\hline $\mathrm{U}$ & 1.2 & 1.1 & 1.1 & 1.2 & 1.1 & 1.1 & 1 & 1.2 & 1.1 \\
\hline $\mathrm{V}$ & 57 & 60 & 80 & 62 & 81 & 80 & 50 & 68 & 87 \\
\hline $\mathrm{Ce}$ & 72 & 78 & 69 & 65 & 73 & 66 & 79 & 82 & 70 \\
\hline Th & 9.8 & 7.2 & 8.5 & 4.7 & 7.3 & 15 & 11 & 2 & 7 \\
\hline
\end{tabular}

schist) and footwall (meta-limestone) (Fig. 8C) are likely due to deformation and alteration processes. There is also the fractionation of LREE relative to HREE; $(\mathrm{La} / \mathrm{Lu})_{\mathrm{N}}$ ratios of metavolcanic, hanging wall and footwall samples are 12.77 (ppm), 12.98 (ppm), and $8.8(\mathrm{ppm})$, respectively (Table 2).

\subsection{Element mobility during mylonitisation}

There are spatial and temporal relationships between the intensity of deformation and element distribution in the metavolcanic host rocks that have experienced various degrees of deformation. The highly deformed ultramylonitic units occur with ore-bearing zones in the inner parts of the Qolqoleh shear zone. High degrees of chloritisation, silicification and sulphidisation in the inner parts of the Qolqoleh shear zone are interpreted as reflecting an increased degree of deformation or changing conditions from weakly deformed rocks (protomy- lonite, more than $50 \mathrm{wt} \%$ porphyroclast) to highly deformed and sheared rocks (ultramylonite, less than $10 \mathrm{wt} \%$ porphyroclast) in the metavolcanic units, whereas low-deformed protomylonitic units are found in the outer parts of the shear zone.

Significant changes in major, trace element, and REE chemistry have occurred during prograde deformation in the Qolqoleh metavolcanics. Ultramylonite rocks are characterised by a decrease in $\mathrm{MgO}$ and $\mathrm{Na}_{2} \mathrm{O}$, and an increase in $\mathrm{SiO}_{2}, \mathrm{CaO}$, and LOI. Relatively immobile components include $\mathrm{P}_{2} \mathrm{O}_{5}$, $\mathrm{K}_{2} \mathrm{O}, \mathrm{Fe}_{2} \mathrm{O}_{3}, \mathrm{Al}_{2} \mathrm{O}_{3}$, and $\mathrm{TiO}_{2}$ (Fig. 9A).

In the Qolqoleh shear zone, $\mathrm{U}, \mathrm{Sc}, \mathrm{Y}$, and $\mathrm{Ba} / \mathrm{Sr}$ remained relatively constant throughout the deformation process, whereas $\mathrm{Rb}$ increased, and $\mathrm{Th}, \mathrm{Ba}$, and $\mathrm{Sr}$ decreased significantly during prograde deformation in the ultramylonites (Fig. 9B, C). In the ultramylonite unit, LREEs were enriched while HREEs remained relatively constant (Fig. 9D).

High gold concentrations in the metavolcanic units correlate with deformation intensity. There 
Table 2. Concentration of major and trace element in the mylonite and ultramylonite metavolcanic units of the Qolqoleh shear zone. MMV: mylonitic metavolcanic; UMV: ultramylonitic metavolcanic; <1 means below detection limit.

\begin{tabular}{|c|c|c|c|c|c|c|c|c|c|}
\hline Sample & GH 2.18 & GH 2.22 & GH 3.16 & GH 4.6 & GH 2.44 & GH 2.47 & GH 4.1 & GH 3.12 & GH 2.40 \\
\hline Sample & ML & ML & CSS & CSS & MMV & MMV & MMV & MMV & MMV \\
\hline La (ppm) & 12.03 & 13.2 & 27.21 & 28.9 & 39.18 & 35.24 & 38.69 & 33.49 & 41.4 \\
\hline $\mathrm{Ce}$ & 21.1 & 16.7 & 57.43 & 58.8 & 74.23 & 69.5 & 69.67 & 54.6 & 73.18 \\
\hline $\operatorname{Pr}$ & 2.2 & 1.7 & 6.5 & 6.7 & 8.93 & 8.31 & 8.26 & 7.54 & 9.17 \\
\hline $\mathrm{Nd}$ & 8.4 & 7.5 & 28 & 28.2 & 37.1 & 35.66 & 37.59 & 32.85 & 39.81 \\
\hline Sm & 1.9 & 1.62 & 5.8 & 5 & 8.3 & 7.3 & 6.9 & 6.2 & 8.2 \\
\hline $\mathrm{Eu}$ & 0.7 & 0.51 & 1.9 & 1.6 & 4.9 & 3.72 & 4.82 & 4.3 & 5.2 \\
\hline $\mathrm{Gd}$ & 2.1 & 1.7 & 5.6 & 5.2 & 8.9 & 6.58 & 6.83 & 5.13 & 7.21 \\
\hline $\mathrm{Tb}$ & 0.4 & 0.24 & 0.75 & 0.6 & 1.46 & 1.02 & 0.98 & 0.78 & 1.14 \\
\hline Dy & 2.1 & 1.4 & 4.1 & 3.6 & 6.72 & 4.51 & 6.04 & 4.8 & 5.74 \\
\hline Ho & 0.4 & 0.32 & 0.7 & 0.6 & 1.3 & 0.9 & 0.97 & 0.81 & 1.1 \\
\hline $\mathrm{Er}$ & 1.15 & 0.9 & 1.6 & 1.3 & 3.39 & 2.56 & 2.59 & 2.2 & 2.91 \\
\hline $\mathrm{Tm}$ & 0.18 & 0.13 & 0.24 & 0.2 & 0.44 & 0.37 & 0.4 & 0.35 & 0.39 \\
\hline $\mathrm{Yb}$ & 1.1 & 0.92 & 1.6 & 1.3 & 3 & 2.19 & 2.75 & 2.3 & 2.93 \\
\hline $\mathrm{Lu}$ & 0.17 & 0.13 & 0.25 & 0.2 & 0.45 & 0.34 & 0.47 & 0.4 & 0.4 \\
\hline$\Sigma \mathrm{REE}$ & 53.93 & 46.97 & 141.68 & 142.2 & 198.3 & 178.2 & 186.96 & 155.75 & 198.79 \\
\hline$(\mathrm{La} / \mathrm{Yb})_{\mathrm{N}}$ & 7.403 & 9.712 & 11.511 & 14.91 & 8.759 & 10.79 & 9.43 & 9.76 & 9.47 \\
\hline$(\mathrm{Eu} / \mathrm{Eu})^{*}$ & 1.077 & 0.944 & 1.024 & 0.964 & 1.752 & 1.649 & 2.158 & 2.344 & 2.092 \\
\hline$(\mathrm{Sm} / \mathrm{Nd})$ & 0.226 & 0.216 & 0.207 & 0.177 & 0.219 & 0.204 & 0.183 & 0.205 & 0.214 \\
\hline Sample & GH 4.19 & GH 4.13 & GH 4.10 & GH 3.6 & GH 3.7 & GH 2.11 & GH 2.29 & GH 2.37 & GH 4.20 \\
\hline Sample & MMV & UMV & UMV & UMV & UMV & UMV & UMV & UMV & UMV \\
\hline $\mathrm{La}(\mathrm{ppm})$ & 31.9 & 75.37 & 60.24 & 50.2 & 52.9 & 69.71 & 58.03 & 60.18 & 87.9 \\
\hline $\mathrm{Ce}$ & 69.48 & 149.6 & 122.54 & 104.6 & 108.34 & 137.6 & 115.7 & 109.6 & 159.33 \\
\hline $\operatorname{Pr}$ & 8.9 & 16.4 & 14.57 & 11.65 & 12.05 & 15.35 & 13.9 & 13.2 & 16 \\
\hline $\mathrm{Nd}$ & 37.8 & 58.12 & 53.2 & 44.18 & 44.37 & 57.12 & 53.12 & 54 & 56.8 \\
\hline $\mathrm{Sm}$ & 8.1 & 9.72 & 9.6 & 8.7 & 8.59 & 9.7 & 9.55 & 9.3 & 9.7 \\
\hline $\mathrm{Eu}$ & 3.8 & 1.43 & 1.7 & 1.37 & 1.82 & 1.54 & 1.61 & 1.33 & 1.2 \\
\hline $\mathrm{Gd}$ & 8.2 & 9.13 & 9.1 & 8.38 & 8.93 & 9.9 & 9.1 & 8.42 & 8.8 \\
\hline $\mathrm{Tb}$ & 1.3 & 1.3 & 1.5 & 1.17 & 1.34 & 1.6 & 1.45 & 1.28 & 1.3 \\
\hline Dy & 5.7 & 8 & 7.88 & 6.25 & 5.93 & 8.26 & 7.94 & 7.08 & 7.5 \\
\hline Ho & 0.9 & 1.3 & 1.34 & 1.14 & 1.09 & 1.39 & 1.37 & 1.22 & 1.4 \\
\hline $\mathrm{Er}$ & 1.9 & 3.69 & 3.6 & 3.31 & 3.1 & 3.76 & 3.81 & 2.9 & 3.3 \\
\hline $\mathrm{Tm}$ & 0.27 & 0.47 & 0.51 & 0.42 & 0.43 & 0.47 & 0.53 & 0.35 & 0.43 \\
\hline $\mathrm{Yb}$ & 2 & 2.87 & 2.97 & 2.59 & 2.56 & 2.83 & 2.4 & 2.71 & 2.5 \\
\hline $\mathrm{Lu}$ & 0.3 & 0.37 & 0.42 & 0.48 & 0.41 & 0.45 & 0.39 & 0.4 & 0.39 \\
\hline$\Sigma$ REE & 180.55 & 337.77 & 289.17 & 244.44 & 251.86 & 319.68 & 278.9 & 271.97 & 356.55 \\
\hline$(\mathrm{La} / \mathrm{Yb})_{\mathrm{N}}$ & 10.69 & 17.61 & 13.6 & 13 & 13.86 & 16.52 & 16.3 & 14.89 & 23.582 \\
\hline$(\mathrm{Eu} / \mathrm{Eu})^{*}$ & 1.433 & 0.466 & 0.558 & 0.493 & 0.638 & 0.48 & 0.53 & 0.462 & 0.398 \\
\hline$(\mathrm{Sm} / \mathrm{Nd})$ & 0.214 & 0.167 & 0.18 & 0.19 & 0.193 & 0.169 & 0.179 & 0.172 & 0.17 \\
\hline
\end{tabular}

is a positive correlation between $(\mathrm{La} / \mathrm{Yb})_{\mathrm{n}}$ ratio and gold content in the mylonitic and ultramylonitic metavolcanics (Fig. 10A). Au concentrations in the mylonite and ultramylonite units are $0.1-0.4$ (ppm) and 1.3-4.5 (ppm), respectively (Tables 2,3). The $\mathrm{Sm} / \mathrm{Nd}$ ratio remains roughly constant during deformation (about 0.2) (Fig. 9D). In the shear zone there are also obvious changes of the $\mathrm{Eu} / \mathrm{Eu}^{*}$ ratio with deformation and alteration intensity $\left(\mathrm{Eu} / \mathrm{Eu}^{*}\right.$ : $1.43-2.34 \mathrm{ppm}$ in mylonite zone, $0.39-0.63 \mathrm{ppm}$ in ultramylonite zone). Mylonitic zones are characterised by a positive Eu anomaly $\left(\mathrm{Eu} / \mathrm{Eu}^{*}>1\right)$ and lower gold content $(\mathrm{Au}=0.1-0.4 \mathrm{ppm})$, whereas ultramylonite units show a negative Eu anomaly $\left(\mathrm{Eu} / \mathrm{Eu}^{*}<1\right)$ and higher gold content $(\mathrm{Au}=1.3-4.5$ ppm) (Tables 2, 3; Figs. 9D, 10B). 

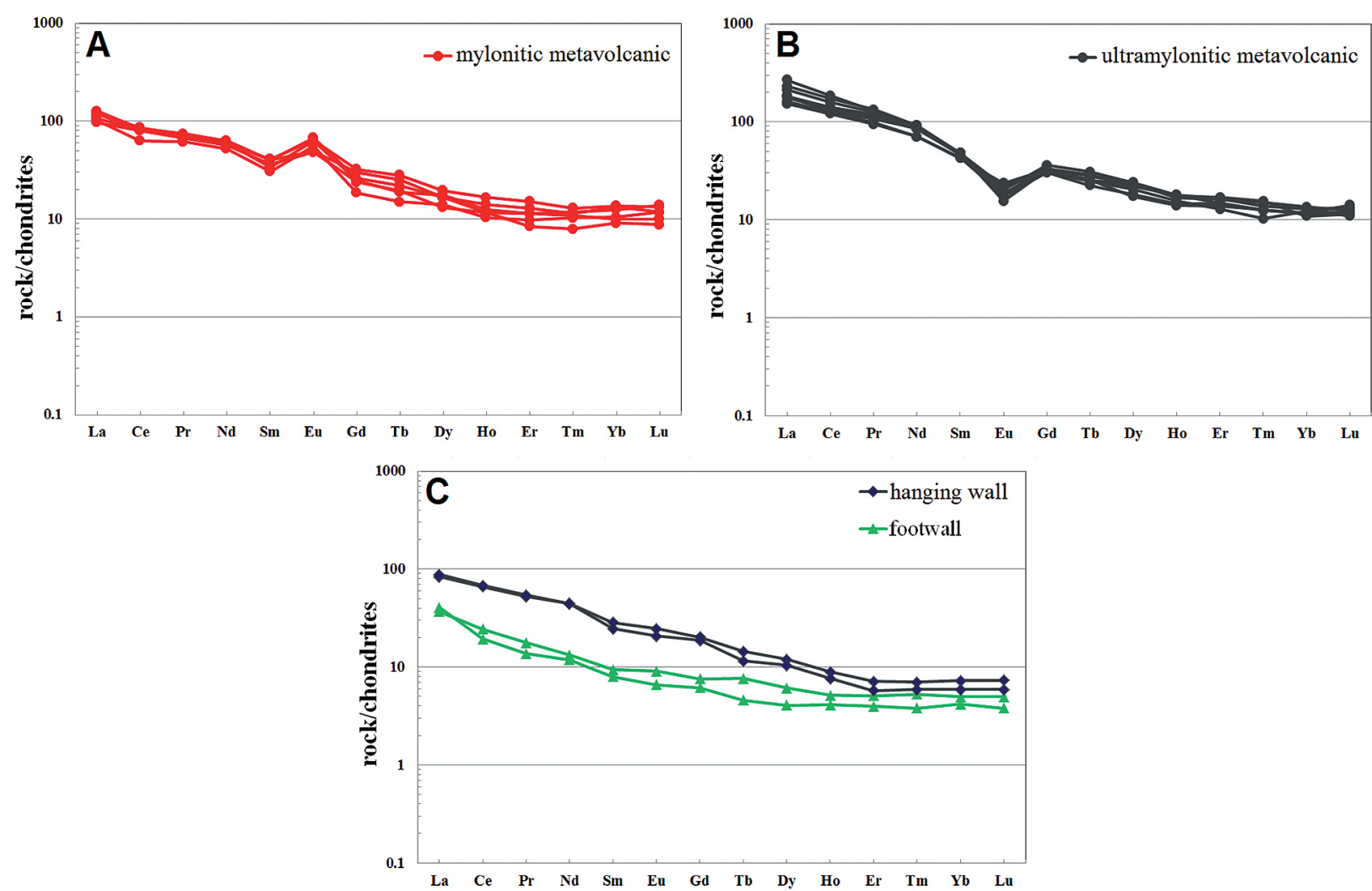

Fig. 8. The REE dispersion pattern in metavolcanic rocks of the Qolqoleh shear zone.

A - Mylonite; B - Ultramylonite; C - Hanging wall (sericite-chlorite schist) and foot wall (meta-limestone). All data are normalised to the Nakamura (1974) chondrite.
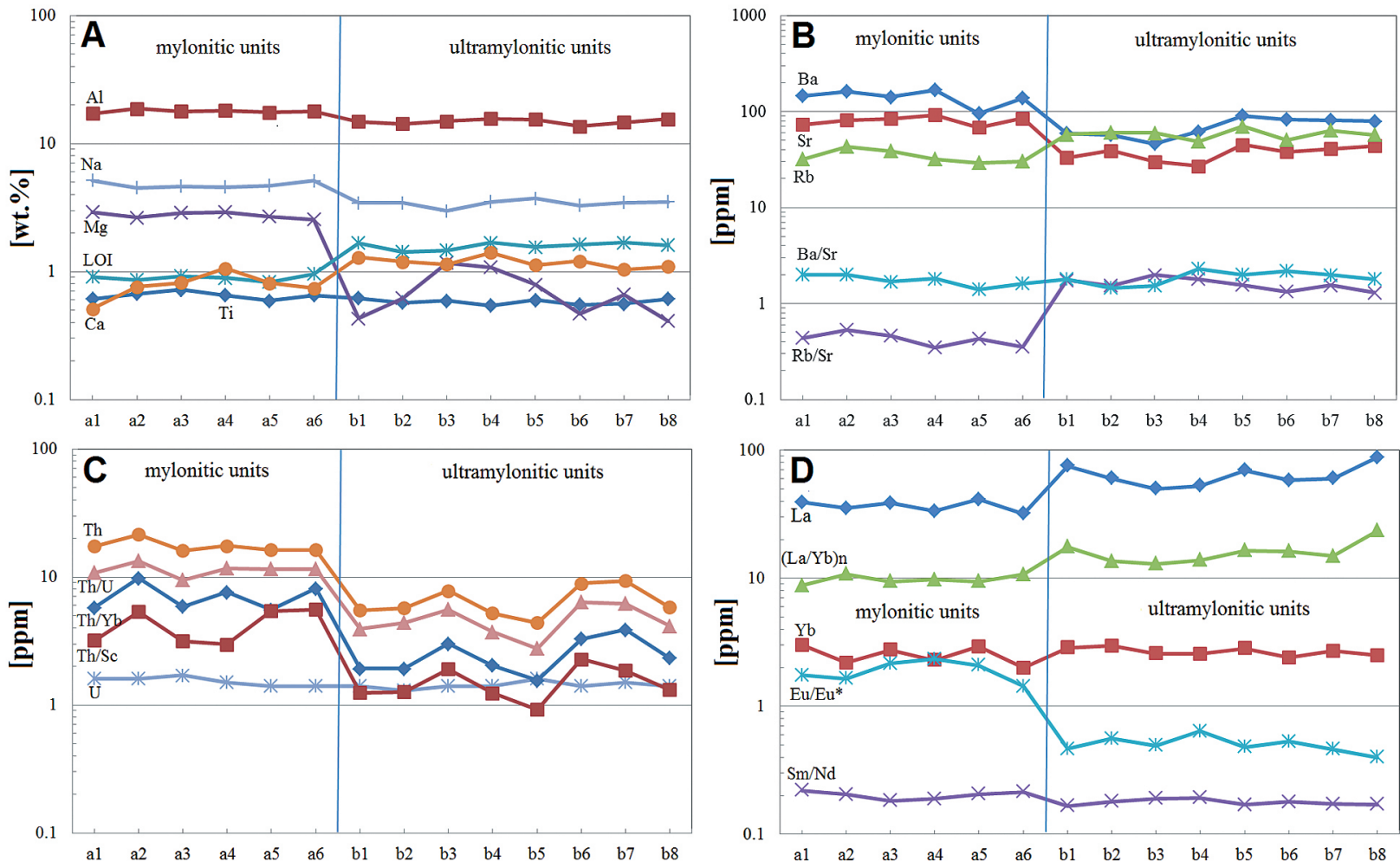

Fig. 9. Distribution patterns: A - major elements; B - HFSE; C - LILE; D - REE in mylonitic and ultramylonitic units. a1-a6 are samples from mylonitic units; b1-b8 from ultramylonitic units. 
Table 3. Concentration of rare earth elements in the hanging wall, foot wall and mineralised shear zone of the Qolqoleh deposit. ML: meta-limestone; CSS: chlorite-sericite schist; MMV: mylonitic metavolcanic; UMV: ultramylonitic metavolcanic; $<1$ means below detection limit.

\begin{tabular}{|c|c|c|c|c|c|c|c|}
\hline Sample & GH 2.44 & GH 2.47 & GH 4.1 & GH 3.12 & GH 2.40 & GH 4.19 & GH 4.13 \\
\hline Sample & MMV & MMV & MMV & MMV & MMV & MMV & UMV \\
\hline Si (\%) & 64.58 & 62.84 & 64.35 & 64.57 & 64.81 & 65.2 & 70.8 \\
\hline $\mathrm{Al}$ & 17.2 & 18.7 & 17.9 & 18 & 17.5 & 17.8 & 14.8 \\
\hline $\mathrm{Fe}$ & 4.8 & 4.92 & 5.02 & 4.75 & 4.65 & 3.25 & 4.31 \\
\hline $\mathrm{Ti}$ & 0.61 & 0.67 & 0.72 & 0.65 & 0.59 & 0.65 & 0.62 \\
\hline $\mathrm{Ca}$ & 0.51 & 0.76 & 0.82 & 1.06 & 0.81 & 0.74 & 1.29 \\
\hline $\mathrm{Na}$ & 5.15 & 4.51 & 4.62 & 4.57 & 4.7 & 5.1 & 3.43 \\
\hline K & 2.9 & 3.13 & 2.64 & 2.57 & 2.47 & 3.18 & 2.81 \\
\hline $\mathrm{Mg}$ & 2.91 & 2.64 & 2.88 & 2.9 & 2.68 & 2.55 & 0.43 \\
\hline $\mathrm{P}$ & 0.31 & 0.45 & 0.42 & 0.43 & 0.39 & 0.37 & 0.32 \\
\hline LOI & 0.91 & 0.86 & 0.92 & 0.89 & 0.83 & 0.96 & 1.67 \\
\hline Co (ppm) & 29.1 & 23.7 & 25.4 & 33.3 & 28.6 & 24.7 & 13.4 \\
\hline $\mathrm{Sc}$ & 5.4 & 4 & 5.1 & 5.9 & 3 & 2.9 & 4.4 \\
\hline Y & 34.7 & 37.4 & 31.5 & 37 & 35.3 & 34.1 & 38.5 \\
\hline $\mathrm{Rb}$ & 32 & 43.2 & 38.6 & 32 & 29.3 & 30.2 & 57.7 \\
\hline $\mathrm{Cr}$ & 28 & 21 & 29 & 18 & 19 & 17 & 13 \\
\hline $\mathrm{Ni}$ & 30 & 16 & 28 & 29 & 19 & 20 & 16 \\
\hline As & 15 & 6.2 & 7.3 & 5.7 & 24 & 1.1 & 50.5 \\
\hline $\mathrm{Pb}$ & 5 & 13 & 9 & 14 & 11 & 8 & 53 \\
\hline $\mathrm{U}$ & 1.6 & 1.6 & 1.7 & 1.5 & 1.4 & 1.4 & 1.4 \\
\hline $\mathrm{Zn}$ & 118 & 109 & 131 & 122 & 94 & 91 & 194.4 \\
\hline $\mathrm{Ag}$ & 0.2 & 0.3 & 0.4 & 0.6 & 0.3 & 0.5 & 1.1 \\
\hline $\mathrm{S}$ & 0.04 & 0.04 & 0.04 & 0.04 & 0.04 & 0.04 & 0.17 \\
\hline $\mathrm{Sb}$ & 0.14 & 0.11 & 0.15 & 0.14 & 0.13 & 0.17 & 0.34 \\
\hline $\mathrm{Bi}$ & 0.05 & 0.05 & 0.04 & 0.06 & 0.06 & 0.05 & 0.18 \\
\hline $\mathrm{Au}$ & 0.3 & 0.4 & 0.1 & 0.3 & 0.3 & 0.2 & 2.9 \\
\hline $\mathrm{Ba}$ & 146 & 161 & 142 & 167 & 95 & 138 & 58.99 \\
\hline $\mathrm{Sr}$ & 73 & 81 & 84 & 92 & 68 & 85 & 33 \\
\hline $\mathrm{Cu}$ & 20.3 & 12.7 & 21.2 & 19.4 & 17.9 & 17.6 & 34.2 \\
\hline Th & 17.3 & 21.4 & 16.1 & 17.5 & 16.2 & 16.2 & 5.5 \\
\hline $\mathrm{Rb} / \mathrm{Sr}$ & 0.438 & 0.533 & 0.459 & 0.347 & 0.43 & 0.355 & 1.748 \\
\hline $\mathrm{Ba} / \mathrm{Sr}$ & 2 & 1.987 & 1.69 & 1.815 & 1.397 & 1.623 & 1.787 \\
\hline $\mathrm{Th} / \mathrm{U}$ & 10.812 & 13.375 & 9.47 & 11.666 & 11.571 & 11.571 & 2.87 \\
\hline $\mathrm{Th} / \mathrm{Yb}$ & 5.766 & 9.771 & 5.854 & 7.6 & 5.529 & 8.1 & 1.916 \\
\hline $\mathrm{Th} / \mathrm{Sc}$ & 3.203 & 5.35 & 3.156 & 2.966 & 5.4 & 5.586 & 1.25 \\
\hline
\end{tabular}

\subsection{Calculation of chemical changes}

Although changes in element concentration with increasing degree of deformation in shear zones have been well documented in the literature (Newman \& Mitra, 1993; Goddard \& Evans, 1995;
Ingles et al., 1999; Hippertt, 1998; Kwon et al., 2009), it is necessary to consider rock densities in order to identify element losses, gains, and changes in rock volume (Condie \& Sinha, 1996). Based on Gresen's equation (1967), Grant (1986) developed an isocon diagram method for mass-balance analysis. As 
Table 3. continued.

\begin{tabular}{|c|c|c|c|c|c|c|c|}
\hline Sample & GH 4.10 & GH 3.6 & GH 3.7 & GH 2.11 & GH 2.29 & GH 2.37 & GH 4.20 \\
\hline Sample & UMV & UMV & UMV & UMV & UMV & UMV & UMV \\
\hline Si (\%) & 71.16 & 71.34 & 70.4 & 69.75 & 72.06 & 70.44 & 70.14 \\
\hline $\mathrm{Al}$ & 14.3 & 14.9 & 15.6 & 15.4 & 13.6 & 14.6 & 15.5 \\
\hline $\mathrm{Fe}$ & 5.33 & 3.9 & 5.18 & 4.5 & 4.53 & 4.3 & 4.62 \\
\hline $\mathrm{Ti}$ & 0.57 & 0.59 & 0.54 & 0.6 & 0.55 & 0.56 & 0.61 \\
\hline $\mathrm{Ca}$ & 1.19 & 1.14 & 1.4 & 1.12 & 1.21 & 1.04 & 1.09 \\
\hline $\mathrm{Na}$ & 3.44 & 2.97 & 3.5 & 3.72 & 3.28 & 3.46 & 3.5 \\
\hline $\mathrm{K}$ & 2.15 & 1.93 & 2.21 & 2.75 & 2.86 & 2.77 & 3 \\
\hline $\mathrm{Mg}$ & 0.62 & 1.17 & 1.08 & 0.79 & 0.47 & 0.66 & 0.41 \\
\hline $\mathrm{P}$ & 0.3 & 0.28 & 0.29 & 0.26 & 0.28 & 0.29 & 0.315 \\
\hline LOI & 1.42 & 1.46 & 1.68 & 1.55 & 1.63 & 1.68 & 1.6 \\
\hline Co (ppm) & 9.3 & 16.1 & 12.2 & 12.6 & 16 & 15.3 & 10.1 \\
\hline $\mathrm{Sc}$ & 4.5 & 4.1 & 4.2 & 4.8 & 3.9 & 5 & 4.4 \\
\hline Y & 37.2 & 34.3 & 37.1 & 33.2 & 35.8 & 35.6 & 33.7 \\
\hline $\mathrm{Rb}$ & 60.1 & 59.5 & 48.6 & 70 & 50.7 & 63.4 & 57 \\
\hline $\mathrm{Cr}$ & 10 & 15 & 12 & 10 & 14 & 14 & 16 \\
\hline $\mathrm{Ni}$ & 14 & 18 & 15 & 14 & 16 & 13 & 18 \\
\hline As & 69.4 & 48.9 & 108 & 123.1 & 55 & 63 & 67.3 \\
\hline $\mathrm{Pb}$ & 56 & 46 & 37 & 29 & 19 & 25 & 24 \\
\hline $\mathrm{U}$ & 1.3 & 1.4 & 1.4 & 1.6 & 1.4 & 1.5 & 1.4 \\
\hline $\mathrm{Zn}$ & 397 & 174 & 280.8 & 266 & 203 & 183 & 228.15 \\
\hline $\mathrm{Ag}$ & 3 & 2.2 & 0.8 & 0.9 & 1.8 & 0.7 & 2 \\
\hline $\mathrm{S}$ & 0.18 & 0.44 & 0.1 & 0.25 & 0.22 & 0.1 & 0.2 \\
\hline $\mathrm{Sb}$ & 0.4 & 0.39 & 0.4 & 0.38 & 0.41 & 0.44 & 0.48 \\
\hline $\mathrm{Bi}$ & 0.17 & 0.22 & 0.18 & 0.23 & 0.2 & 0.18 & 0.24 \\
\hline $\mathrm{Au}$ & 1.3 & 2.7 & 1.4 & 1.9 & 4.5 & 3.1 & 3.6 \\
\hline Ва & 57 & 46 & 62 & 90 & 83 & 81 & 79 \\
\hline $\mathrm{Sr}$ & 39 & 30 & 27 & 45 & 38 & 41 & 44 \\
\hline $\mathrm{Cu}$ & 41.4 & 38.3 & 43.4 & 53.7 & 35.8 & 51.6 & 36.5 \\
\hline Th & 5.7 & 7.8 & 5.2 & 4.4 & 8.9 & 9.3 & 5.8 \\
\hline $\mathrm{Rb} / \mathrm{Sr}$ & 1.541 & 1.983 & 1.8 & 1.555 & 1.334 & 1.546 & 1.295 \\
\hline $\mathrm{Ba} / \mathrm{Sr}$ & 1.461 & 1.533 & 2.296 & 2 & 2.184 & 1.975 & 1.795 \\
\hline $\mathrm{Th} / \mathrm{U}$ & 4.384 & 5.571 & 3.714 & 2.75 & 6.357 & 6.2 & 4.142 \\
\hline $\mathrm{Th} / \mathrm{Yb}$ & 1.919 & 3.011 & 2.031 & 1.554 & 3.284 & 3.875 & 2.32 \\
\hline Th/Sc & 1.266 & 1.902 & 1.238 & 0.916 & 2.282 & 1.86 & 1.318 \\
\hline
\end{tabular}

Kwon et al. (2009) indicated, identification of 'immobile' elements is an important step because improper choice will lead to erroneous results.

The AI-Ti-Fe isocon (Grant, 1986, 2005; Condie \& Sinha, 1996) is used to investigate element behaviour in the Qolqoleh shear zone. The best-fit isocon closely approximates the constant Al-Ti-Fe isocon, but this is distinct from the isocons corresponding to either constant volume or constant mass. Based on the Al-Ti-Fe isocon diagram of metavolcanics, $\mathrm{Si}, \mathrm{S}, \mathrm{Ca}, \mathrm{As}, \mathrm{Zn}, \mathrm{Cu}, \mathrm{Au}, \mathrm{Ag}, \mathrm{Sb}, \mathrm{Bi}, \mathrm{Pb}, \mathrm{Rb}$, LOI, and LREE show enrichment, whereas $\mathrm{Sr}, \mathrm{Ba}, \mathrm{Na}$, 

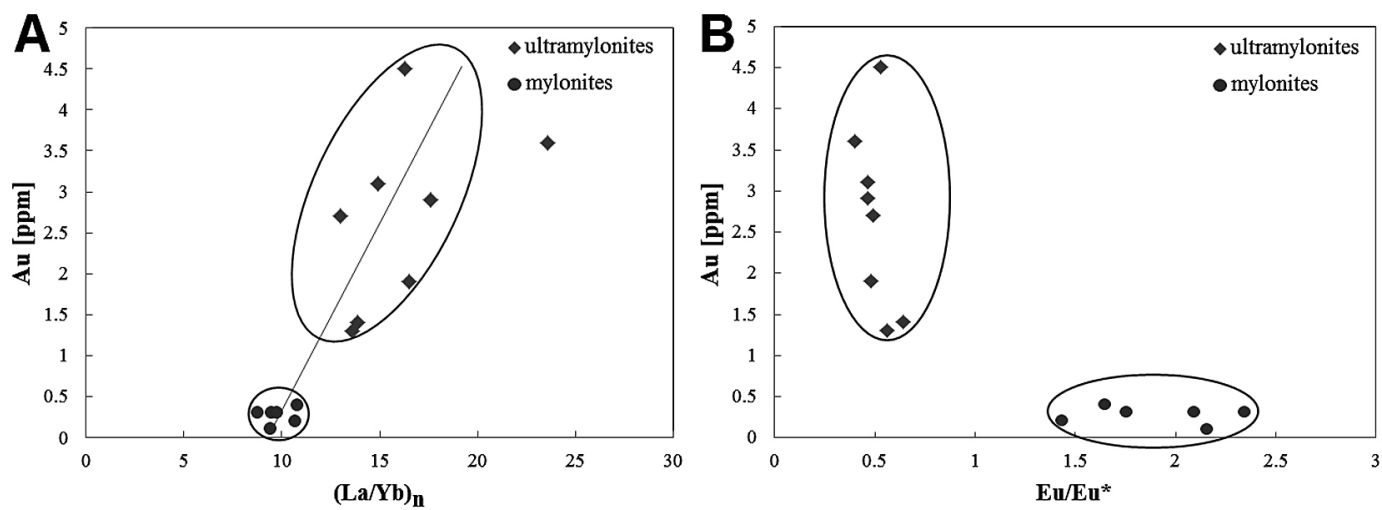

Fig. 10. Chondrite-normalised scatter diagrams for $\mathrm{Au} v s(\mathrm{La} / \mathrm{Yb})_{\mathrm{n}}$ and $\mathrm{Au} v s \mathrm{Eu} / \mathrm{Eu}^{*}$ in altered mylonitic metavolcanics and ultramylonitic metavolcanics.
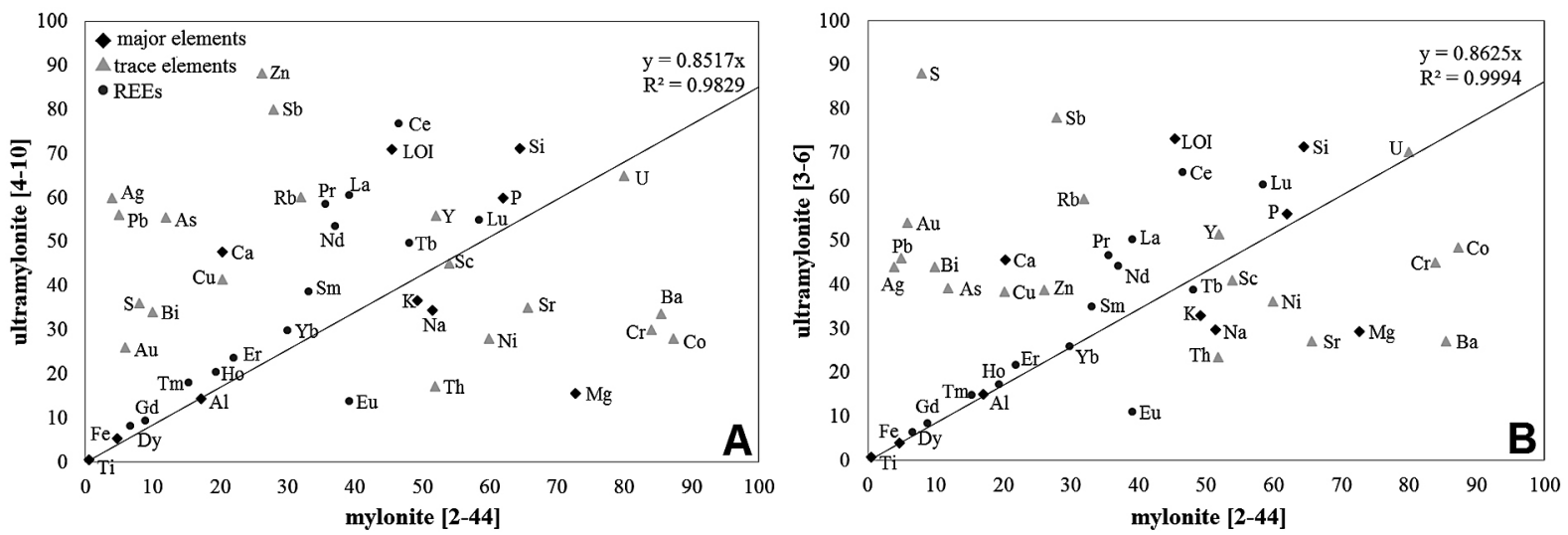

Fig. 11. Isocon diagrams in mylonites vs ultramylonites of the Qolqoleh shear zone. Sample numbers in square brackets

$\mathrm{Eu}, \mathrm{Mg}$, Th, $\mathrm{Cr}, \mathrm{Co}$, and Ni show depletion during prograded/prograding deformation (Fig. 11). Relatively immobile elements include $\mathrm{Al}, \mathrm{Ti}, \mathrm{Fe}, \mathrm{Sc}, \mathrm{Y}$, $\mathrm{K}, \mathrm{U}, \mathrm{P}$ and HREE (except $\mathrm{Eu}$ ). An increase in $\mathrm{Si}$, S, $\mathrm{Zn}, \mathrm{Pb}$, and $\mathrm{Cu}$ is consistent with sulphide, sericite and silicic alterations in the Qolqoleh shear zone. It seems that these elements precipitated during hydrothermal alteration by Si-bearing fluids, which contained bi-sulphide complexes in highly deformed zones. The occurrence of pyrite and galena corresponds to the enrichment of $\mathrm{Au}, \mathrm{Sb}, \mathrm{As}$, and $\mathrm{Ag}$ in this unit. The increase in Ca and LOI is related to hydrous minerals (e.g. muscovite and epidote) in ultramylonites. $\mathrm{Mg}$, $\mathrm{Sr}, \mathrm{Na}$, and $\mathrm{Ba}$ depletion is followed by decomposition of biotite and feldspar minerals during mylonitisation (Sinha et al. 1986; Condie and Sinha 1996). In addition, other elements such as $\mathrm{Cr}, \mathrm{Ni}$, and $\mathrm{Co}$ have been depleted with increasing deformation intensity. Based on Rollinson (1993), these elements are relatively immobile, and may be mobile on a local scale (McCuaig \& Kerrich 1998).

\subsection{Classification of the Qolqoleh shear zone}

Based on changes in volume or mass during deformation, shear zones are classified into three types (O'Hara, 1988; O'Hara \& Blackburn, 1989; Selverstone et al., 1991, Bailey et al., 1994; Kwon et al., 2009): 1) Isovolume types indicating that no volume change occurred in the shear zones; these types of shear zones are commonly related to subvertical strike-slip setting (e.g., Kerrich et al., 1980); 2) Volume-loss types indicating that elements decreased during progression from protomylonite to ultramylonite; these shear zones are related to compressional tectonic settings (O'Hara, 1988; Dipple et al., 1990), and 3) Volume-gain types which are related to transtensional tectonic settings (Glazner \& Bartley, 1991; Bailey et al. 1994; Hippertt, 1998; Kwon et al., 2009).

As illustrated in Fig. 12B, in the volume-loss shear zones with an increased degree of deformation, both $\mathrm{Na}_{2} \mathrm{O}$ and $\mathrm{SiO}_{2}$ decrease, whereas in the IVG shear zone, $\mathrm{Na}_{2} \mathrm{O}$ decreases while $\mathrm{SiO}_{2}$ increas- 

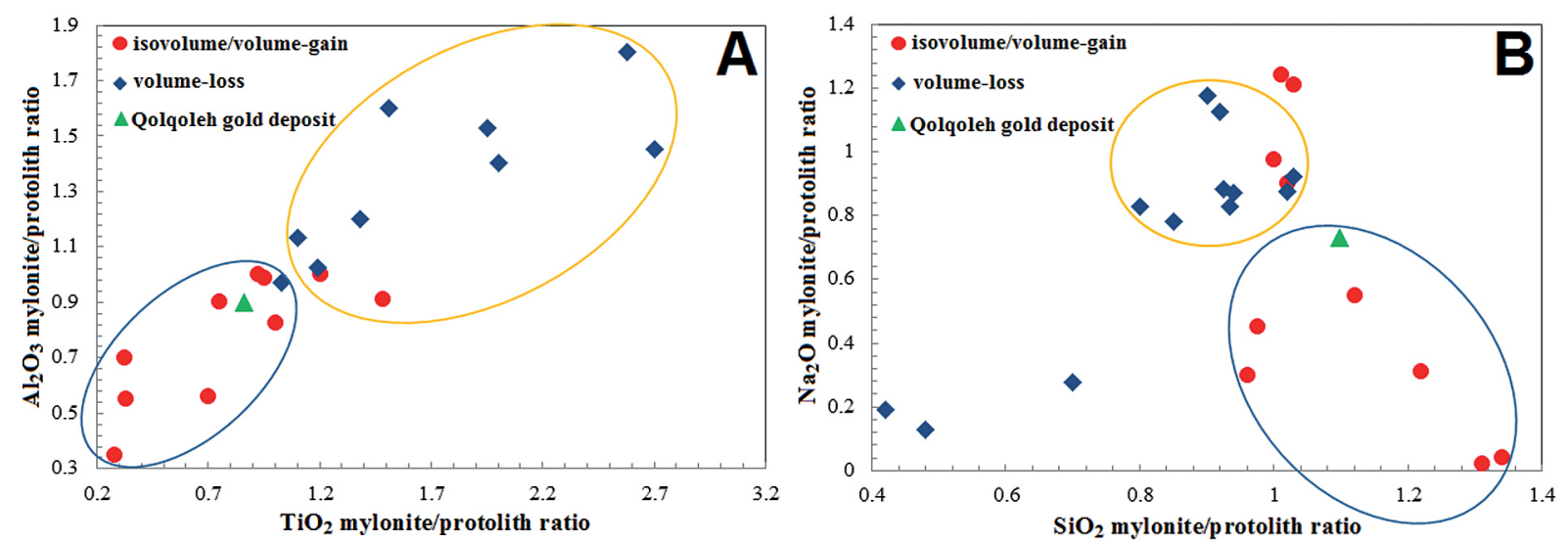

Fig. 12. Two plots of concentration ratios of mylonite/protolith in deformed matavolcanics from shear zones. Red- and blue-marked samples from Candie \& Sinha (1996), green-marked ones are average samples from the Qolqoleh gold deposit (the present study).

es. It seems that the decrease in $\mathrm{Na}_{2} \mathrm{O}$ and $\mathrm{SiO}_{2}$ in the volume-loss shear zones is related to feldspar destruction because of passage of large amounts of fluid through shear zones (Glazner \& Bartley 1991; Condie \& Sinha 1996). In contrast, the IVG ultramylonites often show petrographic evidence of syntectonic silicification. Each type of shear zone can be classified by using the $\mathrm{Al}_{2} \mathrm{O}_{3}-\mathrm{TiO}_{2}$ diagram (Fig. 12A). These oxides have been enriched in the volume-loss shear zones; whereas the IVG shear zones have been depleted (Condie \& Sinha 1996).

The Qolqoleh shear zone is compared with other types of shear zones in the $\mathrm{SiO}_{2}-\mathrm{Na}_{2} \mathrm{O}$ and $\mathrm{TiO}_{2}-$ $\mathrm{Al}_{2} \mathrm{O}_{3}$ diagrams (Fig. 12). By an increased degree of deformation or changing conditions from protomylonite to ultramylonite, $\mathrm{SiO}_{2}$ increases while $\mathrm{Na}_{2} \mathrm{O}$ remains relatively constant (Fig. 12B). Correspondingly, in the $\mathrm{Al}_{2} \mathrm{O}_{3}-\mathrm{TiO}_{2}$ diagram, by an increased deformation intensity, $\mathrm{TiO}_{2}$ and $\mathrm{Al}_{2} \mathrm{O}_{3}$ decrease (Fig. 12A). Based on these diagrams, the shear zone studied is classified as Isovolume-Gain (IVG) type.

\section{Fluid inclusion}

\subsection{Fluid inclusion petrography}

Based on the number of phases present at room temperature (Shepherd et al., 1985) and their microthermometric features, three fluid inclusion types were recognised: Type I monophase aqueous inclusions (V), Type II two-phase (liquid+vapour) aqueous inclusions, and Type III three-phase carbonic-aqueous inclusions (liquid water-liquid
$\mathrm{CO}_{2}$-vapour $\mathrm{CO}_{2}$ ). Type II is the most abundant of the three inclusion types. In all types of inclusions, daughter mineral phases were absent.

\subsubsection{Monophase aqueous inclusion (Type I)}

These types of fluid inclusions occur in both quartz veins and are characterised by one phase (liquid or gas) at room temperature. They are transparent, have a spherical shape and vary in size (3 and $8 \mu \mathrm{m})$ (Fig. 13A, B).

\subsubsection{Aqueous two-phase fluid inclusions (Type II)}

These inclusions are seen in both concordant gold-bearing quartz-sulphide veins, and barren quartz-calcite veins and are characterised by a vapour bubble in an aqueous liquid at room temperature, transparency and low relief. In these inclusions, vapour bubble occupies $25-40 \%$ of the total inclusion volume. Morphologically, they show variations from negative crystal to spherical, rectangular, elongate, oval or irregular forms, and their sizes range from 4 to $18 \mu \mathrm{m}$ (Fig. 13C, D). The primary to pseudosecondary inclusions occur as isolated single or clusters of randomly aligned inclusions.

\subsubsection{Carbonic-aqueous inclusions (Type III)}

These primary inclusions generally are 5-15 $\mu \mathrm{m}$ in size and consist of three phases, two immiscible liquids (liquid $\mathrm{CO}_{2}+$ liquid water) and a small $\mathrm{CO}_{2}$ vapour bubble at room temperature occupying $30 \%$ of the total inclusion volume. These inclusions may be irregular, oval or rounded in shape, but are found only in gold-bearing quartz-sulphide veins and veinlets (Fig. 13E, F). 

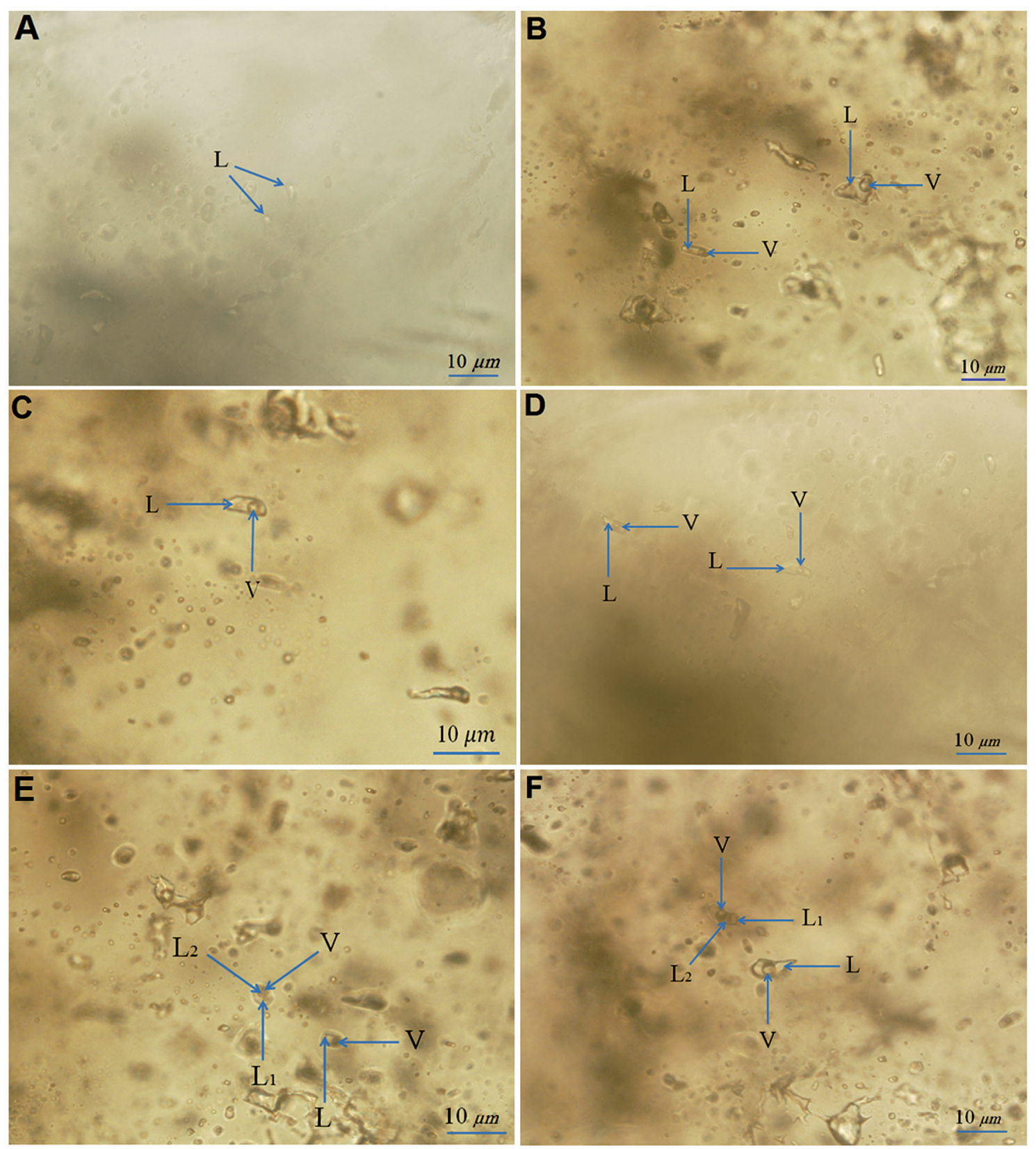

Fig. 13. Petrographic images of fluid inclusion.

A - Type I monophase aqueous fluid inclusion; B-D - Type II two-phase aqueous fluid inclusion (L+V); E, F - threephase carbonic aqueous inclusions (Aqueous $+\mathrm{LCO}_{2}+\mathrm{VCO}$ ).

\subsection{Fluid inclusion microthermometry}

Initial melting of $\mathrm{CO}_{2}$ phase $\left(\mathrm{T}_{\mathrm{M} \mathrm{CO} 2}\right), \mathrm{CO}_{2}$ homogenisation $\left(\mathrm{T}_{\mathrm{H} \mathrm{CO} 2}\right)$, and final melting of clathrate $\left(\mathrm{T}_{\mathrm{M} \text { Clath }}\right)$ for $\mathrm{CO}_{2}$-bearing inclusions, as well as final melting of ice ( $\left.\mathrm{T}_{\mathrm{M} \text { ICE }}\right)$ and total homogenisation $\left(\mathrm{T}_{\mathrm{H} \text { Total }}\right)$ for aqueous inclusions were measured (Table 4).

Data obtained from the microthermometric measurements and volume fraction estimates of the phases (for the purpose of transform melting and homogenisation temperatures and optical volume fraction estimates into bulk compositions and densities) were processed using the software packages Fluids (Bakker, 1999) and clathrates (Bakker, 1997). Calculations of salinity for aqueous and carbonic aqueous inclusion, fractions of compositions, density of carbonic liquid and bulk fluid, and bulk molar volume of fluid inclusions were made using the FLINCOR program by Brown (1989). The FLINCOR software and Bodnar (1983) diagram were used for density calculation. For pressure estimates in two- 
Table 4. Summary of fluid inclusion data for gold-bearing quartz-sulphide and barren quartz-calcite veins from the Qolqoleh gold deposit.

\begin{tabular}{|c|c|c|c|c|c|c|c|c|}
\hline Inclusion type & $\begin{array}{l}\text { Number of } \\
\text { inclusions }\end{array}$ & $\begin{array}{l}\text { Size } \\
{[\mu \mathrm{m}]}\end{array}$ & $\begin{array}{c}\mathrm{TM}_{\mathrm{CO} 2} \\
{\left[{ }^{\circ} \mathrm{C}\right]}\end{array}$ & $\begin{array}{c}\mathrm{T}_{\mathrm{M} \text { Clath }} \\
{\left[{ }^{\circ} \mathrm{C}\right]}\end{array}$ & $\begin{array}{c}\mathrm{T}_{\mathrm{HCO} 2} \\
{\left[{ }^{\circ} \mathrm{C}\right]}\end{array}$ & $\begin{array}{l}\mathrm{T}_{\mathrm{MICE}} \\
{\left[{ }^{\circ} \mathrm{C}\right]}\end{array}$ & $\begin{array}{c}\mathrm{T}_{\mathrm{H} \text { Total }} \\
{\left[{ }^{\circ} \mathrm{C}\right]}\end{array}$ & $\begin{array}{c}\text { Salinity } \\
{[\mathrm{wt} \% \mathrm{NaCl}]}\end{array}$ \\
\hline $\begin{array}{l}\text { type II-a } \\
\text { (aqueous two- } \\
\text { phase) }\end{array}$ & 72 & $3-8$ & & & & -9.7 to -3 & 204 to 255 & 4.85 to 13.62 \\
\hline $\begin{array}{l}\text { type II-b } \\
\text { (aqueous two- } \\
\text { phase) }\end{array}$ & 43 & $4-18$ & & & & -16.2 to -11.2 & 335 to 386 & 15.17 to 19.58 \\
\hline $\begin{array}{l}\text { type III } \\
\text { (carbonic- } \\
\text { aqueous) }\end{array}$ & 30 & $5-15$ & -58.2 to -56.4 & 1.3 to 4.7 & 20.4 to 25.3 & & 345 to 386 & 9.43 to 13.98 \\
\hline
\end{tabular}

phase aqueous inclusions, the equation states of $\mathrm{H}_{2} \mathrm{O}$ in P-T projection were used (Diamond, 2003).

\subsubsection{Aqueous two-phase inclusions (Type II)}

About $78 \%$ of the inclusions studied are aqueous inclusions $(\mathrm{L}+\mathrm{V})$. Based on $\mathrm{T}_{\mathrm{H}^{\prime}}$ this type of inclusions is classified into two groups: a) Those with low-intermediate $\mathrm{T}_{\mathrm{h}}\left(205-255^{\circ} \mathrm{C}\right.$, with a sharp peak at $235^{\circ} \mathrm{C}$; Fig. $14 \mathrm{~A}$ ). The $\mathrm{T}_{\mathrm{M} \mathrm{ICE}}$ of this type fluid inclusion ranges from -3 to $-9^{\circ} \mathrm{C}$ (Fig. 14B), indicating salinities between 5-13 wt \% $\mathrm{NaCl}$ equivalent (with a high frequency at 11-12 wt $\% \mathrm{NaCl}$ equivalent; Fig. 14C) and bulk fluid densities between 0.84 and $0.96 \mathrm{gr} / \mathrm{cm}^{3}$ (Fig. 15). This type of fluid inclusion is the most abundant; and b) High $\mathrm{T}_{\mathrm{h}}$ from 335 to $385^{\circ} \mathrm{C}$ (Fig. 14A) and $\mathrm{T}_{\mathrm{M} \mathrm{ICE}}$ from -11 to $-16^{\circ} \mathrm{C}$ (Fig. 14B), indicating salinities between 15 and $19 \mathrm{wt} \%$ $\mathrm{NaCl}$ equivalent (with a sharp peak at $16 \mathrm{wt} \% \mathrm{NaCl}$ equivalent; Fig. 14C). Bulk fluid density ranges between 0.79 to $0.86 \mathrm{~g} / \mathrm{cm}^{3}$ (Fig. 15).

\subsubsection{Carbonic-aqueous inclusions (Type III)}

Total homogenisation $\left(\mathrm{T}_{\mathrm{H} \text { Total }}\right)$ into the liquid phase was observed between 345 and $385^{\circ} \mathrm{C}$, with a mode at $365^{\circ} \mathrm{C}$ (Fig. 14A). These inclusions show $\mathrm{T}_{\mathrm{MCO} 2}$ ranging from -56.4 to $-58.3^{\circ} \mathrm{C}$. Homogenisation of the carbonic phase $\left(\mathrm{T}_{\mathrm{H} \mathrm{CO} 2}\right)$, typically to a liquid, was in a narrow range of temperatures between 20.4 and $25.3^{\circ} \mathrm{C}$. Melting of the $\mathrm{CO}_{2}$ clathrate $\left(\mathrm{T}_{\mathrm{M}}\right.$ Clath) in the presence of $\mathrm{CO}_{2}$ liquid occurred between 1.3 and $4.7^{\circ} \mathrm{C}$ (Fig. 14B) indicating a salinity of 9-13 wt $\% \mathrm{NaCl}$ equivalent (Fig. 14C). Bulk fluid densities range between 0.89 and $0.93 \mathrm{gr} / \mathrm{cm}^{3}$ (Fig. 15).

\subsection{Pressure condition}

Trapping pressure can be estimated from the fluid inclusions trapped under immiscible or boiling con- ditions or if an independent trapping temperature is known (Brown \& Hagemann, 1995). Estimates of trapping pressures for ore-forming fluids in Type II and Type III fluid inclusions were determined using the Touret and Dietvorst (1983) and Diamond (2003) approaches. The highest homogenisation temperature $\left(385^{\circ} \mathrm{C}\right)$ is considered as the minimum trapping temperature. The minimum pressure is in the range of 1.6 to $2 \mathrm{kbar}$ (Fig. 16), suggesting that the Qolqoleh gold deposit must have formed at a depth of at least $7.4 \mathrm{~km}$. This depth and the brittle-ductile nature of the ore zones indicate that Qoqoleh constitutes a mesozonal subtype gold deposit (e.g., Groves et al., 1998; Goldfarb et al., 2005).

\section{Oxygen and hydrogen isotopes}

The gold-bearing quartz-sulphide veins hosted in altered-mineralised metavolcanics and discordant quartz-calcite veins in barren or low-mineralised metavolcanics were analysed for oxygen isotopes (Table 5). Hydrogen isotope analyses were performed on fluid inclusions released from samples of both quartz vein types (Table 5).

Oxygen isotopic compositions of hydrothermal water in equilibrium with quartz were estimated utilising extrapolation of the fractionation formula from Clayton et al. (1972). The fractionation factors were calculated using the mean value of the total homogenisation temperatures of fluid inclusions, plus pressure-corrected temperatures as discussed below. Assuming a quartz-sulphide vein deposition temperature of $369-385^{\circ} \mathrm{C}$ (based upon the fluid inclusion study outlined above), calculated values for $\delta^{18} \mathrm{O}_{\text {fluid }}$ approximately ranged between $7 \%$ and $9 \%$. In addition, the $\delta \mathrm{D}$ values are from $-40.23 \%$ o to -35.27 . This is indicative of an isotopically heavy crustal fluid and likely of little involve- 

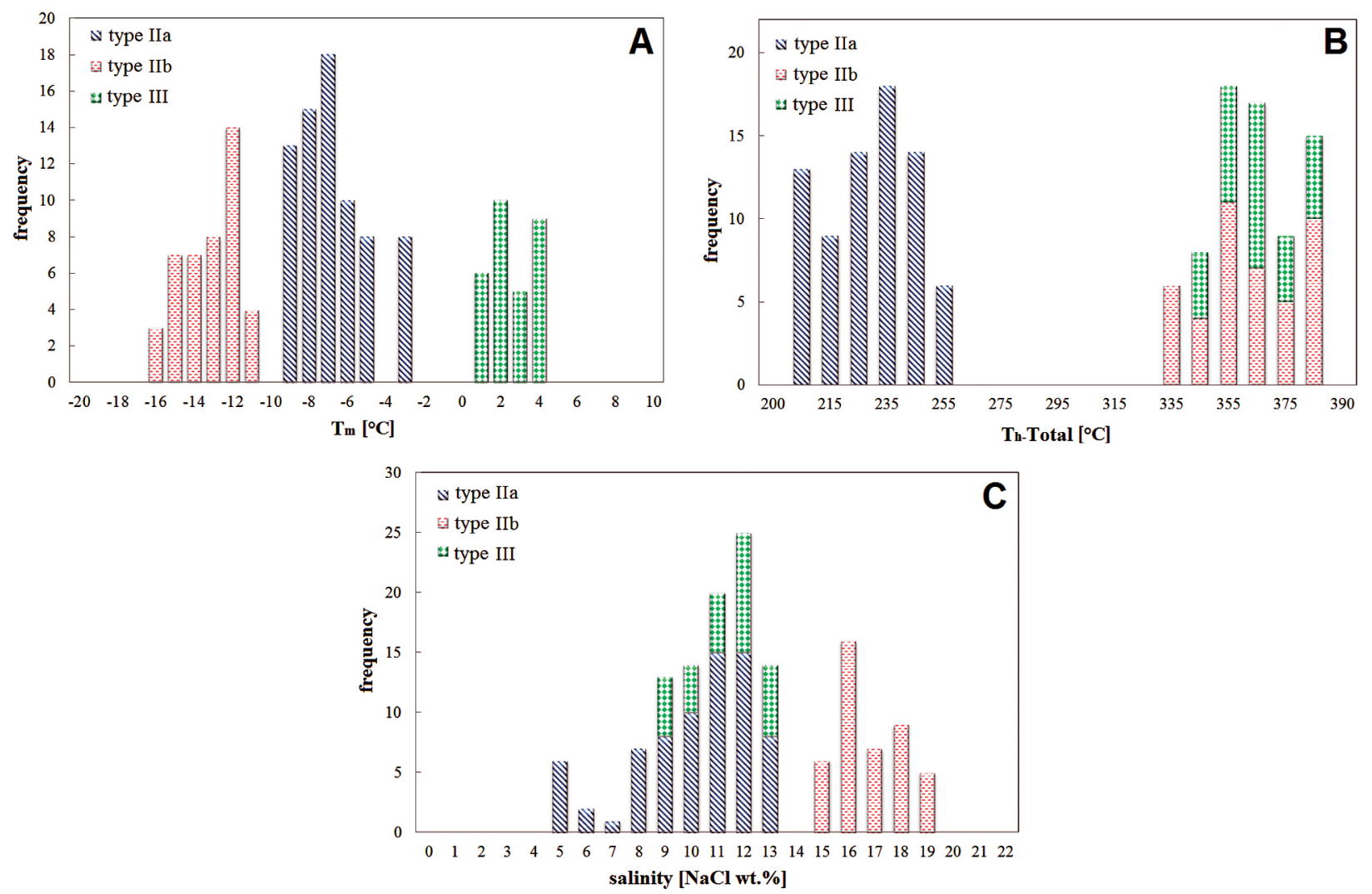

Fig. 14. Fluid inclusion results of quartz veins from the Qolqoleh gold deposit.

A - Homogenisation temperature; B - Melting point of ice and of clathrate; C - Salinity of aqueous fluid inclusions (type IIa, type IIb) and type III carbonate-aqueous fluid inclusions.

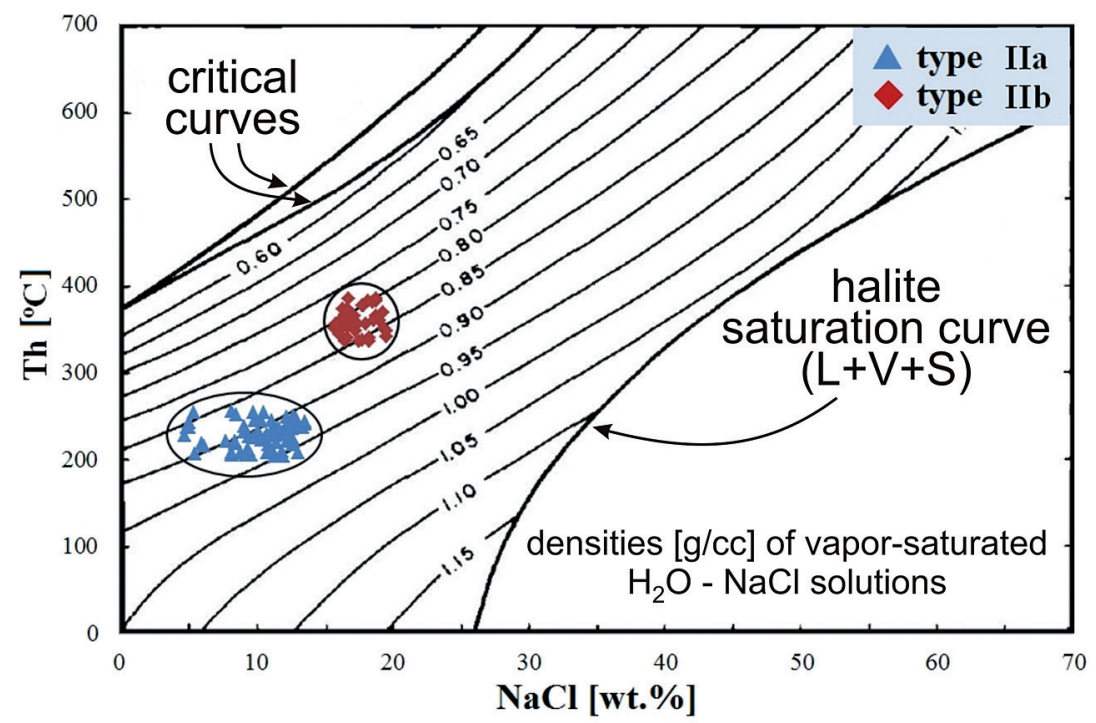

Fig. 15. Salinity-temperature diagram showing density of aqueous fluid inclusions (boundaries after Bodnar, 1983).

ment of meteoric fluid. Sample locations occupy the field of metamorphic fluids (most orogenic gold deposits), and imply involvement of isotopically metamorphic waters during ore formation (Fig. 20). Using temperatures of $249-250^{\circ} \mathrm{C}$, the calculated $\delta \mathrm{O}^{18}$ values of water in equilibrium with the late-stage discordant quartz-calcite veins varies from $-5.31 \%$ o to $-3.35 \%$, while the $\delta \mathrm{D}$ values range from $-5.65 \%$ o to $-75.31 \%$. These values are clearly lower than those of early-stage quartzsulphide-gold veins, and are close to the meteoric water line. 
Table 5. Oxygen and hydrogen isotope compositions (SMOW) for quartz from gold-bearing and barren veins of the Qolqoleh deposit. $\delta^{18} \mathrm{O}_{\text {water }}$ values are calculated using extrapolation of the fractionation formula from Clayton et al. (1972).

\begin{tabular}{ccccc}
\hline Sample & $\mathrm{T}_{\mathrm{H}}\left({ }^{\circ} \mathrm{C}\right)$ & $\delta \mathrm{D} \%$ o & $\delta^{18} \mathrm{O} \%$ & $\delta^{18} \mathrm{O}_{\text {water }}$ \\
\hline Q 3.32 & 369 & -35.270 & 12.8 & 8.010 \\
Q 3.6 & 385 & -40.230 & 11.6 & 7.210 \\
Q 2.24 & 250 & -75.310 & 5.55 & -3.350 \\
Q 2.9 & 249 & -95.653 & 3.6 & -5.310 \\
\hline
\end{tabular}

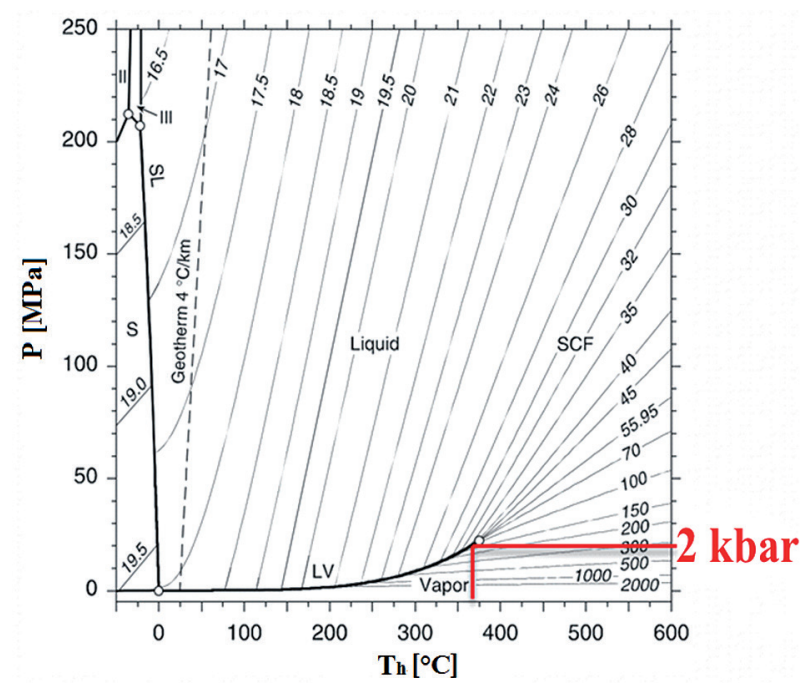

Fig. 16. Temperature-pressure diagram showing pressure during trapping of ore-bearing fluids (boundaries after Diamond, 2003).

\section{Discussion}

\subsection{Hydrothermal alteration and REE mobility}

Numerous investigations have been carried out on mobility, fractionation, solubility, and behaviour of REE in hydrothermal and shear zone environments. Rolland et al. (2003) believed that the mobility of REE in the shear zone depended of $\mathrm{pH}, \mathrm{fO}_{2}$, the fluid composition, $\mathrm{P}-\mathrm{T}$ conditions, and the availability of $\mathrm{CO}_{3}^{2-}, \mathrm{PO}_{4}^{2-}, \mathrm{SO}_{4}^{2-}, \mathrm{F}^{-}$, and $\mathrm{H}^{-}$ligands in the fluid. In addition, sulphate complexes (Felsche \& Herrmann, 1978), and carbonic fluids (Wendlandt \& Harrison, 1979) can control the mobility of REE and preferential fractionation of LREE vs HREE. Furthermore, solubility of the REE in hydrothermal systems can be related to carbonate and fluoride complexes (Wood, 1990; Haas et al., 1995). Elevated concentrations of REE may reflect availability of complexing agents, mainly $\mathrm{SO}_{4}{ }^{2-}$ for LREE and F-for HREE (Rolland et al., 2003). Various ligands may carry and precipitate REE in response to changes in fluid chemistry, due to fluid/rock interaction or fluid mixing processes. The mineralogy and composition of fluid inclusion phases can be used to determine the existence of complexing agents in the hydrothermal fluid. At Qolqoleh, the existence of $\mathrm{CO}_{2}$-bearing fluid inclusions and carbonate-pyrite association in veins and shear zones indicates the presence of carbonate and sulphur complexes in the mineralising fluid. It seems that LREE enrichment may not be associated with the presence of $\mathrm{CO}_{2}$ alone (Rolland et al., 2003) and, possibly, sulphate complexes were involved in LREE enrichment because they preferentially form complexes with LREE (Lewis et al., 1997).

It seems that LREE enrichment at Qolqoleh resulted from circulation of $\mathrm{SO}_{4}^{2-}$ and $\mathrm{CO}_{2}$-bearing fluids due to regional metamorphism in the shear zones. Likewise, the weak fractionation in modified REE patterns (LREE to HREE) could be related to regional effects of metamorphism (greenschist facies) and sericite-chlorite alteration. Geochemical studies show HREE depletion in metavolcanics of the Qolqoleh shear zone. In some cases, HREE enrichment is attributed to decreasing temperature and availability of $\mathrm{OH}$ and F-bearing fluids, which could form complexes preferentially with HREE (Haas et al., 1995). HREE depletion may relate to leaching of HREE by F-bearing fluids during increased intensity of hydrothermal alteration at Qolqoleh.

Rolland et al. (2003) thought that changes in REE concentration were related to fluid/rock interaction in shear zones; hence mobility of REE depends of the stability of REE-bearing minerals and thus, directly to fluid. Therefore, considering the low fractionation degree of the REE pattern, the absence of a Eu anomaly and any evidence of fluid/rock interaction in hanging wall and footwall are indicative of any alteration and deformation.

\subsection{Eu anomaly}

In order to investigate Eu behaviour in the metavolcanics, some samples were taken from ultramylonite and mylonite units. The results indicate the existence of positive and negative $\mathrm{Eu}$ anomalies in the mylonite and ultramylonite units, respectively (Fig. 17A, B). It seems that the positive and negative $\mathrm{Eu}$ anomalies are correlated with the intensity degree of plagioclase alteration. 

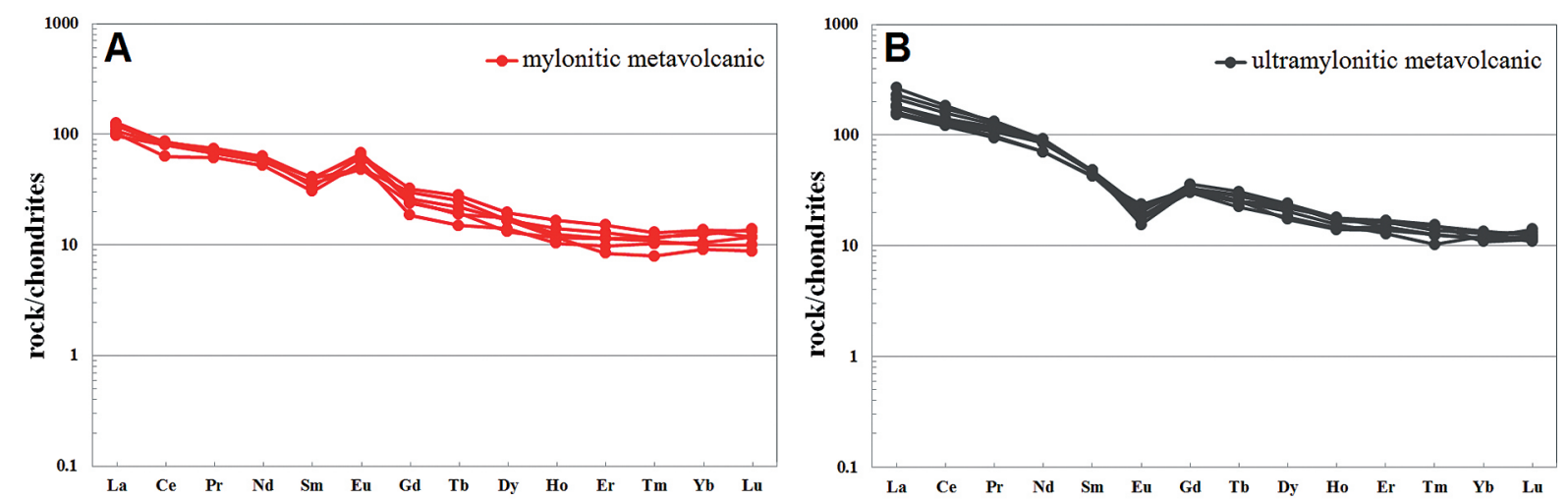

Fig. 17. A - Negative Eu anomaly in highly deformed metavolcanics (ultramylonitic); B - Positive Eu anomaly in less deformed metavolcanics (protomylonitic-mylonitic).

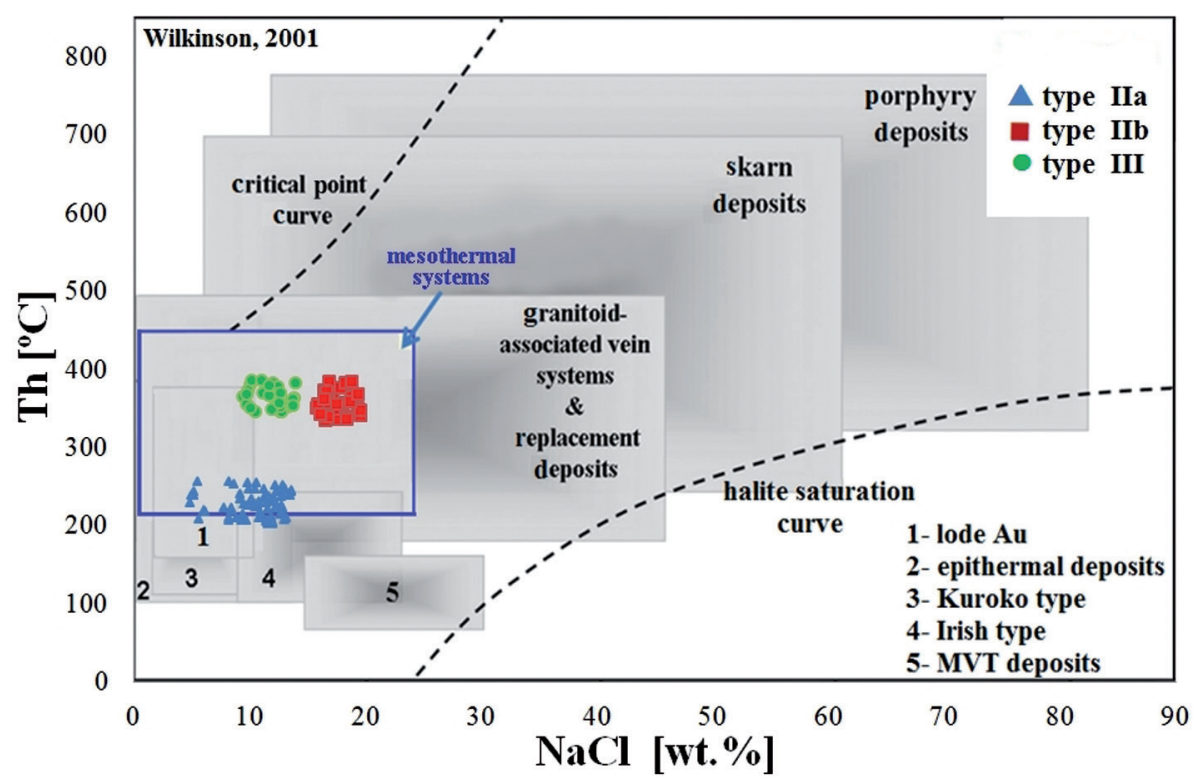

Fig. 18. Temperature-salinity diagram for various types of ore deposits (Wilkinson, 2001). Data from the Qolqoleh deposit plot in the mesothermal deposit field.

The Eu anomalies observed in the REE pattern could be connected to the widespread existence distribution of plagioclase. In mylonitised samples, there is some weak plagioclase alteration to sericite. This could account for the release of some REE from feldspar and mica and REE establishment in secondary sericite. Subsequently, a slight increase in the Eu anomaly (Fig. 17A) and LREE enrichment than HREE are the only chemical changes observed (Schnetzler et al., 1970). Geochemical studies conducted on strongly altered and deformed rocks have shown the role of these agents in the REE distribution pattern.

Increased intensity of deformation in ultramylonite units led to development of microfractures and ores in the rocks, which facilitated fluid flow and ultimately resulted in an increase of the fluid/rock ratio. This process strengthened/boosted the hydrothermal alteration effect involving feldspars. Therefore, to consider plagioclase as the major carrier and the main source of Eu depletion in these units conforms to the progress of the alteration-deformation process and increasing degree of plagioclase decomposition (Fig. 17B).

\subsection{Classification of Qolqoleh gold deposit}

The chemistry of vein-forming fluids is remarkably similar in orogenic gold ores of all ages (Groves et al., 2000). Detailed fluid inclusion studies in most greenstone-hosted orogenic Au deposits have shown ore-fluid to contain consistently about $5 \mathrm{~mol} \% \mathrm{CO}_{2}$, low salinity $\mathrm{H}_{2} \mathrm{O}-\mathrm{CO}_{2} \pm \mathrm{CH}_{4} \pm \mathrm{N}_{2}$ fluid with low $\mathrm{H}_{2} \mathrm{~S}$, traces of $\mathrm{CH}_{4}$ and $\mathrm{N}_{2}$, and low $\mathrm{fO}_{2}$ (Groves et al., 1998, 2000; Pirajno, 2009). Orogenic 
gold deposits differ from other gold-rich deposit groups in the same terranes, which are formed at uniformly shallow depths (normally $5 \mathrm{~km}$ below surface) from highly saline low- $\mathrm{CO}_{2}$ fluids (e.g. VHMS and porphyry-style deposits) or acidic low$\mathrm{CO}_{2}$ fluids (epithermal deposits) and which, characteristically, are either base-metal $( \pm \mathrm{Sn} \pm \mathrm{Mo})$ and/or silver-enriched (Groves et al., 2000).

The hydrothermal environment of ore-forming fluids at Qolqoleh, based on homogenisation temperature and salinity, was mesothermal (Fig. 18), which, based on a minimum pressure of 1.7-2 kbar, formed at 6.3 to $7.4 \mathrm{~km}$ depth (Fig. 16). Based on the tectonic setting of the Qolqoleh area and depth of formation, this deposit is classified as an orogenic gold deposit of the mesozonal subtype (e.g., Groves et al., 1998; Goldfarb et al., 2005). The petrographic and microthermometric characteristics of the fluid inclusions in this type of deposits include small size (>20), low salinity $(2<\mathrm{wt} \% \mathrm{NaCl}<15)$ (Roedder, 1984), homogenisation temperature from 300 to $475^{\circ} \mathrm{C}$ (Groves et al., 1998), $\mathrm{H}_{2} \mathrm{O}-\mathrm{CO}_{2} \pm \mathrm{CH}_{4} \pm \mathrm{N}_{2}$-rich fluid inclusions, densities from 0.8 to $1.5 \mathrm{gr} / \mathrm{cm}^{3}$ (Groves et al., 1998; Goldfarb et al., 2005), gold mineralisation in the brittle-ductile regimes that have undergone greenschist facies metamorphism, and trapping depth for ore-forming fluids (depth of formation) between 6-12 km (Groves et al., 1998). All of these criteria apply to the Qolqoleh gold deposit. Based upon fluid inclusions data, total homogenisation temperature in the gold-bearing quartz-sulphide veins occurred between $335-385^{\circ} \mathrm{C}$ (Fig. $14 \mathrm{~A})$, and the main values of density of ore-forming fluids, pressure and depth of mineralisation are $0.79-0.93 \mathrm{gr} / \mathrm{cm}^{3}$ (Fig. 15), 1.7-2 kbar, 6.3-7.4 $\mathrm{km}$ (Fig. 16), respectively. In addition, the presence of aqueous-carbonic inclusions with low salinity (indicator of metamorphic environments) demonstrates the obvious similarity of fluid inclusions of the Qolqoleh brittle-ductile shear zone to ore-forming fluids in orogenic gold deposits.

\subsection{Source of ore-forming fluids}

Fluids responsible for gold mineralisation can originate from various sources including magmatic-hydrothermal (Pattrick et al., 1988; De Ronde et al., 2000), metamorphic dehydration (Goldfarb et al., 1991; McCuaig \& Kerrich, 1998), meteoric water (Nesbitt et al., 1989; Hagemann et al., 1994), $\mathrm{CO}_{2}$-rich fluids liberated from the mantle (Cameron, 1988, 1989; Colvine, 1989), or a mixed source of deep-seated magmatic and shallow meteoric water (Zhang et al., 2005).
In the orogenic gold deposits, $\delta^{18} \mathrm{O}$ values of hydrothermal fluids are about 5-8 \% for Archean greenstone belts, and about $2 \%$ higher for Phanerozoic gold-bearing belts (Groves et al. 1998). These deposits also show that the $\delta \mathrm{D}$ values range from $-30 \%$ o to $-80 \%$ o (Guilbert \& Park 1997). This range is comparable with metamorphic (Burrows \& Spooner 1989) and magmatic fluids (So \& Yun 1997). Such data from selected orogenic gold deposits are plotted out of the so-called meteoric water (Hagemann et al. 1994) or reaction with organic matter (Goldfarb et al. 1997). Results of stable isotope studies demonstrate the role of different fluids in the genesis of quartz-sulphide and quartz-calcite veins during the evolution of the Qolqoleh gold deposit. The $\delta^{18} \mathrm{O}$ and $\delta \mathrm{D}$ values for water in equilibrium with the quartz-sulphide veins plot within the field of metamorphic fluids and orogenic gold deposits (Fig. 19). This suggests that the Qolqoleh ore-mineralising fluids originated from devolatilisation during deep metamorphism. Devolatilisation of these units occurred synchronously with, or postdates, the development of penetrative (ductile) structures such as shear zones, folds, regional penetrative fabrics, and during overprinting brittle deformation (Aliyari et al., 2009).

The $\delta^{18} \mathrm{O}$ and $\delta \mathrm{D}$ values of water in equilibrium with the late-stage quartz-calcite veins indicate a meteoric water source (Fig. 19). Such fluids are nearly neutral, with relatively low $S$ contents, temperatures of about 200 to $350^{\circ} \mathrm{C}$ and low salinity (Kesler, 2005). These criteria are consistent with the mineralogy and fluid inclusion data of the Qolqoleh deposit. Faulting, microfracturing, and jointing provided suitable pathways for fluid migration during the final stages of deformation.

\subsection{Tectonic model for Qolqoleh ore formation}

Almost all gold occurrences that have been discovered recently in the northwestern SSZ, formed during the late Zagros orogeny complex. Previous studies (Aliyari et al., 2009; Niromand et al., 2011) indicated that the pre-accretionary gold enrichment in this part of the Zargos shear zone could define a more favourable metal source reservoir for later hydrothermal fluids, and thus, suggest a higher prospectivity for a world-class orogenic gold deposit in the northwestern SSZ. Qolqoleh and other Saqqez-Sardasht-Piranshahr gold occurrences are located in a highly deformed area, close to the eastern edge of the Zagros shear zone and along the structurally complex contact between the Precam- 


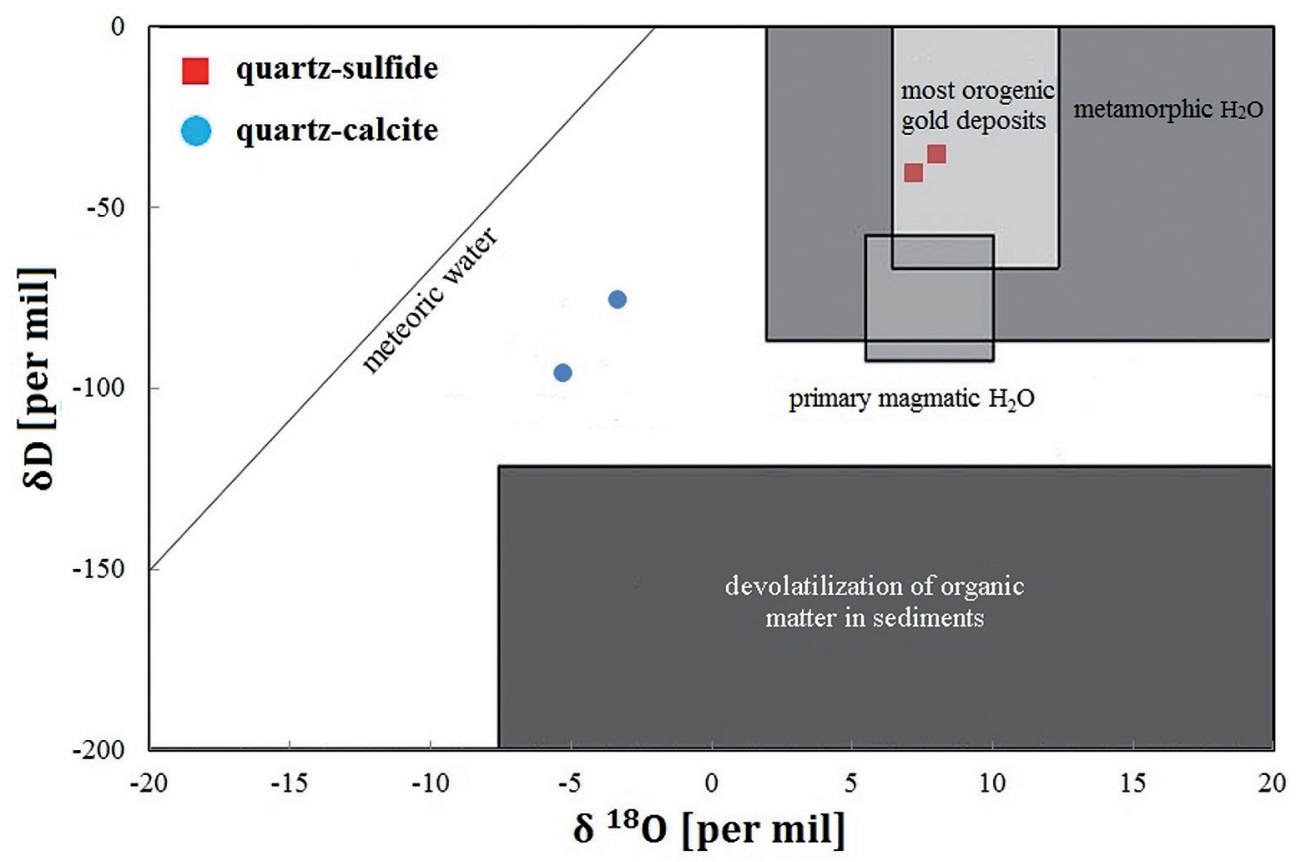

Fig. 19. $\delta^{18} \mathrm{O}$ vs $\delta \mathrm{D}$ diagram for water in equilibrium with quartz-sulphide and quartz-calcite veins.

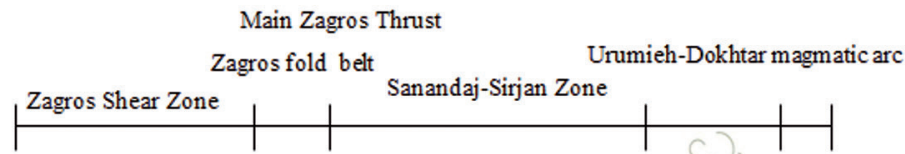

\section{Oligocene to Miocene}

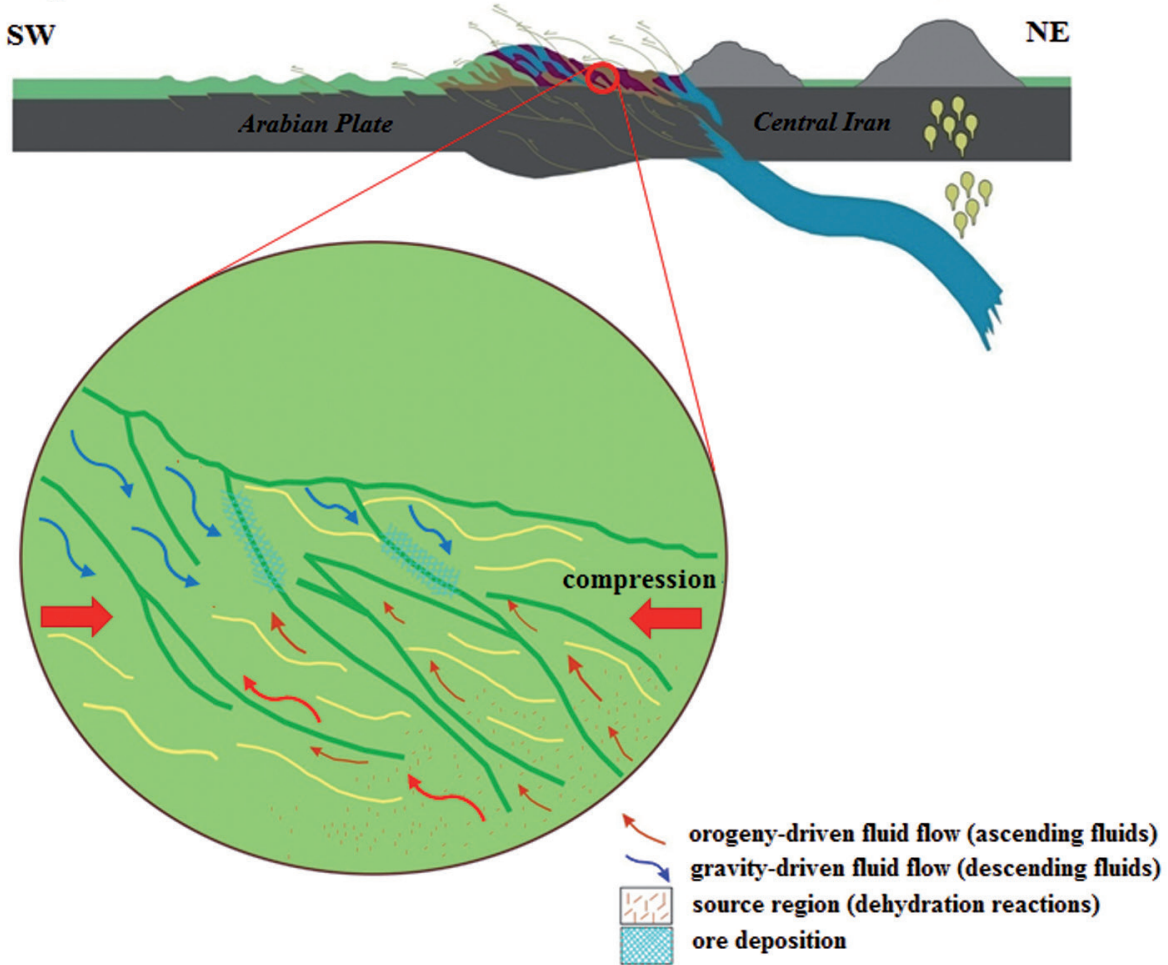

Fig. 20. Conceptual model of gold deposition mechanism in the Qolqoleh shear zone. Extensional conditions which prevailed after orogeny provided an environment conducive to the mobility and migration of ore-bearing fluids. Also circulation of shallow meteoric fluids may have helped in fluid mixing and resulted in deposition of sulphides and gold. 
brian basement and the Cretaceous accreted oceanic rocks.

Gold mineralisation in the Qolqoleh deposit, which is controlled by ductile and brittle deformation of the volcano-sedimentary rocks, is developed within structurally controlled dilational fractures. Tectonic setting, isotopic composition and fluid inclusion data suggest that collision-related devolatilisation process during deep metamorphism of the Cretaceous volcano-sedimentary sequences to be the main mechanism of ore fluid formation. Aliyari et al. (2009) argued that metamorphic devolatilisation was focused in boundary areas between the S-SE- and N-NE- trending structural domains and constituted the key factor for the metallogenesis of the orogenic gold deposits in the Sanandaj-Sirjan zone. Accordingly, the volcano-sedimentary sequences progressively deformed during the collision of the Arabian continental and central Iranian microcontinental plates in the Late Jurassic-Early Cretaceous. This compressional tectonic regime led to the formation of a ductile shear zone consisting of protomylonite, mylonite, and ultramylonite. Nevertheless, no significant gold mineralisation occurred during the ductile stage due to a lack of favourable channel ways and insufficient heat energy to drive fluid circulation (Aliyari et al., 2009), until transformation of the tectonic regime from compressional to extensional. During this stage, the uplift of the region induced changes from ductile to brittle regime which in turn caused the distribution of cross structures, fractures, faults, and vein systems. Therefore, changes from compressional to extensional conditions, in association with increasing geothermal gradients (emplacement of intrusive bodies), caused the flow of ascending metamorphic fluids and circulation of gravity-driven fluids (meteoric water), which led to fluid mixing and deposition of sulphide minerals with gold (Fig. 20).

\section{Conclusions}

The Qolqoleh gold deposit is a ductile to brittle-orogenic deposit within the northwestern part of the Sanandaj-Sirjan metamorphic belt. The rocks in the vicinity of the Qolqoleh deposit predominately consist of Cretaceous volcano-sedimentary sequences of phyllite, meta-limestone, cholorite-sericite schist, metavolcanic and Oligocene granitoid rocks that have undergone greenschist facies metamorphism. Geochemical studies indicate that gold mineralisation occurred in the sulphide-quartz veins.
Fluid-rock interaction during evolution of the Qolqoleh shear zone is commonly associated with changes in major and trace elements chemistry. REE behaviour during hydrothermal fluid-rock interaction in the various rock units shows the existence of strong REE enrichment in metavolcanics of shear zone relative to hanging wall (chlorite-sericite schist) and foot wall (meta-limestone). It seems that LREE enrichment resulted from circulation of $\mathrm{SO}_{4}{ }^{2-}$ and $\mathrm{CO}_{2}$-bearing fluids due to episodes of regional metamorphism in the shear zones.

Our investigations demonstrate the spatial and temporal relationships between deformation intensity and element distribution in and adjacent to mylonites and ultramylonites in the Qolqoleh shear zone. Mass transfer calculations indicate that chemical changes that occurred during deformation include enrichment of $\mathrm{Si}, \mathrm{S}, \mathrm{Ca}, \mathrm{As}, \mathrm{Zn}, \mathrm{Cu}, \mathrm{Au}, \mathrm{Ag}$, $\mathrm{Sb}, \mathrm{Bi}, \mathrm{Pb}, \mathrm{Rb}$, LOI, and LREE, and depletion of $\mathrm{Sr}$, $\mathrm{Ba}, \mathrm{Na}, \mathrm{Eu}, \mathrm{Mg}, \mathrm{Th}, \mathrm{Cr}, \mathrm{Co}$, and Ni. Relatively immobile components include $\mathrm{Al}, \mathrm{Ti}, \mathrm{Fe}, \mathrm{Sc}, \mathrm{Y}, \mathrm{K}, \mathrm{U}, \mathrm{P}$, and HREE (except Eu).

A negative correlation was observed between the $\mathrm{Eu} / \mathrm{Eu}^{*}$ ratio and gold content, whereas a positive correlation was found between the $(\mathrm{La} / \mathrm{Yb})_{\mathrm{n}}$ ratio and gold content in mylonitic and ultramylonitic metavolcanics. Mylonitic zones are characterised by a positive $\mathrm{Eu}$ anomaly $\left(\mathrm{Eu} / \mathrm{Eu}^{*}>1\right)$ and lower gold content, whereas ultramylonite units show a negative Eu anomaly $\left(\mathrm{Eu} / \mathrm{Eu}^{*}<1\right)$ and higher gold content.

Two types of quartz veins are found in the Qolqoleh shear zone: concordant mylonitic quartz-sulphide veins, and discordant mylonitic quartz-carbonate veins. Gold mineralisation occurs in the former. Fluid inclusions microthermometry shows two distinct ranges of homogenisation temperatures $\left(205-255^{\circ} \mathrm{C}\right.$ and $\left.335-385^{\circ} \mathrm{C}\right)$. Both microthermometric and stable isotope data indicate that two types of fluids were involved in the mineralisation, 1) a high-temperature, moderately saline and deep metamorphic fluid which formed quartz-sulphide-gold mineralisation, and 2) low-temperature, low-salinity, meteoric fluid responsible for latestage quartz-calcite mineralisation.

The Qolqoleh gold mineralisation is of the orogenic type (like other deposits: Mute, Kherapeh and Karavian in the Sanandaj-Sirajn Zone). The Cretaceous volcano-sedimentary sequences of the Saqqez-Sardasht-Piranshahr zone which hosts gold deposits was progressively deformed during the continental collision of the Arabian Plate with the Iranian microcontinent. This deformation was accompanied by plutonism and greenschist facies metamorphism. No significant gold mineralisation 
occurred during ductile deformation and compressional tectonic regime due to a lack of favourable channel ways and insufficient heat energy to drive fluid circulation (Aliyari et al., 2009). Major gold mineralisation took place when the tectonic regime transformed from compressional to extensional system. In this zone, changes from compressional to extensional regimes, combined with increasing geothermal gradients (emplacement of intrusive bodies), induced remobilisation of considerable amounts of metamorphic fluids and finally deposited sulphide minerals with gold mineralisation.

\section{Acknowledgments}

The authors are grateful to the research committee of Shiraz University for supporting this project, and acknowledge Dr Farhang Aliyari for constructive comments and Kimberlee Sparks for help in carrying out stable isotope analyses at Cornell University. Thanks are extended also to Dr Farid Moore (Shiraz University) for useful comments.

\section{References}

Agard, P., Omrani, J., Jolivet, L. \& Mouthereau, F., 2005. Convergence history across Zagros (Iran): constraints from collisional and earlier deformation. International Journal of Earth Sciences 94, 401-419.

Alavi, M., 1994. Tectonics of the Zagros orogenic belt of Iran: new data and interpretations. Tectonophysics 229, 211-238.

Alavi, M., 2004. Regional stratigraphy of the Zagros folded-thrust belt of Iran and its proforeland evolution. American Journal of Science 304, 1-20.

Alavi, M., 2007. Structures of the Zagros fold-thrust belt in Iran. American Journal of Science 307, 10641095.

Aliyari, F., Rastad, E. \& Zengqian, H., 2007. Orogenic gold mineralization in the Qolqoleh gold deposit, Northwestern Iran. Resource Geology 57, 262-289.

Aliyari, F., Ebrahim, R., Mohammad, M. \& Greg, B.A., 2009. Geology and geochemistry of D-O-C isotope systematics of the Qolqoleh gold deposit, Northwestern Iran: Implications for ore genesis. Ore Geology Reviews 36, 306-314.

Aliyari, F., Ebrahim, R., Goldfarb, R. \& Sharif, j.A., 2012. Geochemistry of hydrothermal alteration at the Qolqoleh gold deposit in the northern part of the Sanandaj-Sirjan metamorphic belt, northwestern Iran. Journal of Geochemical Exploration DOI: 10.1016/j.gexplo.2012.02.001.
Arvin, M., Pan, Y., Dargahi, S., Malekizadeh, A. \& Babaei, A., 2007. Petrochemistry of the Siah-Kuh granitoid stock southwest of Kerman, Iran: implications for initiation of Neotethys subduction. Journal of Asian Earth Sciences 30, 474-489.

Azizi, H. \& Moinevaziri, H., 2009. Review of the tectonic setting of Cretaceous to Quaternary volcanism in northwestern Iran. Journal of Geodynamics 47, 167-179.

Baharifar, A., Moinevaziri, H., Bellon, H. \& Pique, A., 2004. The crystalline complexes of Hamadan (Sanandaj-Sirjan Zone, western Iran): metasedimentary Mesozoic sequences affected by late Cretaceous tectono-metamorphic and plutonic events. Comptes Rendus Geoscience 336, 14431452.

Bailey, C.M., Simpson, C. \& De Paor, D.G., 1994. Volume loss and tectonic flattening strain in granitic mylonites from the Blue Ridge province, central Appalachians. Journal of Structural Geology 16, 1403-1416.

Bakker, R.J., 1997. Clathrates: computer programs to calculate fluid inclusion $\mathrm{V}-\mathrm{X}$ properties using clathrate melting temperatures. Computer Geosciences 23, 1-18.

Bakker, R.J., 1999. Optimal interpretation of microthermometrical data from fluid inclusions: thermodynamic modeling and computer programming. Ruprecht-Karls University, Heidelberg, 50 pp. (in German)

Bau, M., 1991. Rare-earth elements mobility during hydrothermal and metamorphic fluid-rock interaction and the significance of the oxidation state of Europium. Chemical Geology 93, 219-230.

Berberian, M., 1976. Seismotectonic Map of Iran, 1:2500000. Geological Survey of Iran.

Berberian, F. \& Berberian, M., 1981. Tectono-plutonic episodes in Iran. [In:] Gupta, H.K. \& Delany, F.M. (Eds), Zagros-Hijndu Kush-Himalaya geodynamic evolution. American Geophysical Union, Geodynamic Series 3, 5-32.

Berberian, M. \& King, G.C.P., 1981. Towards a paleogeography and tectonic evolution of Iran. Canadian Journal of Earth Sciences 18, 210-26.

Berberian, M., 1983. The Southern Caspian: A Compressional Depression Floored by a Trapped, Modified Oceanic Crust. Canadian Journal of Earth Sciences 20, 163-83.

Berberian, M., 1995. Master blind thrust faults hidden under the Zagros folds: active basement tectonics and surface morphotectonics. Tectonophysics 241, 193-224.

Bierlein, F.P., Hughes, M., Dunphy, J., McKnight, S., Reynolds, P.R. \& Waldron, H.M., 2001. Trace element geochemistry, 40Ar/39Ar ages, Sm-Nd 
systematics and tectonic implications of mafic-intermediate dykes associated with orogenic lode gold mineralisation in central Victoria, Australia. Lithos 58, 1-31.

Bodnar, R.J., 1983. A method of calculating fluid inclusion volumes based on vapor bubble diameters and P-V-T-X properties on inclusion fluid. Economic Geology 78, 535-542.

Brown, P.E., 1989. FLINCOR: A microcomputer program for the reduction and investigation of fluid-inclusion data. American Mineralogist Journal 74, 1390-1393.

Brown, P.E. \& Hagemann, S.G., 1995. MacFlincor and its application to fluids in Archean lodegold deposits. Geochimica et Cosmochima Acta 59, 3943-3952.

Burrows, D.R. \& Spooner, E.T.C ., 1989. Pb isotope geochemistry of the Silidor and Launay gold deposits; implications for the source of Archean $\mathrm{Au}$ in the Abitibi Subprovince. Economic Geology 88, 1722-1730.

Cameron, E.M., 1988. Archean gold: relation to granulite formation and redox zoning in the crust. Geology 16, 109-112.

Cameron, E.M., 1989. Scouring of gold from the lower crust. Geology 17, 26-29.

Clayton, R.N., O,Neil, L.R. \& Mayeda, T.K., 1972. Oxygen isotope exchange between quartz and water. Journal of Geophysical Research 77, 30573067.

Colvine, A.C., 1989. An empirical model for the formation of Archean gold deposits: Products of final cratonization of the Superior Province, Canada, [In:] Keys, R.R., Ramsay, W.R.H. \& Groves, D.I. (Eds), The Geology of Gold Deposits: The Perspective in 1988. Economic Geology Monograph $6,37-53$

Condie, K.C. \& Sinha, A.K., 1996. Rare earth and other trace element mobility during mylonitization: a comparison of the Brevard and Hope Valley shear zones in the Appalachian mountains, USA. Journal of Metamorphic Geology 14, 213-226.

De Ronde, C.E.J., Faure, K., Bray, C.J. \& Whitford, D.J., 2000. Round Hill shear zone-hosted gold deposit, Macraes flat, Otago, New Zealand: evidence of a magmatic ore fluid. Economic Geology 95, 1025-1048.

Diamond, L.W., 2003. Systematics of H2O inclusions. [In:] Samson, I., Anderson, A. \& Marshall, D. (Eds) Fluid Inclusions: Analysis and Interpretation. Mineralogical Association of Canada, Short Course 32, 55-79.

Dickin, A.P., 1988. Evidence for limited REE leaching from the Roffna Gneiss, Switzerland - a dis- cussion of the paper by Vocke et al. Contributions to Mineralogy and Petrology 99, 273-275.

Dipple, G.M., Wintsch, R.P. \& Andrews, M.S., 1990. Identification of the scales of differential element mobility in a ductile shear zone. Journal of Metamorphic Geology 8, 645-661.

Eftekhar-Nezhad, J., 1973. The Mahabad Quadrangle Map, 1:250000. Geological Survey and Mineral Exploration of Iran.

Eftekhar-Nezhad, J., 1981. Tectonic division of Iran with respect to sedimentary basins. Journal of Iranian Petroleum Society 82, 19-28 (in Farsi).

Felsche, J. \& Herrmann, A.G., 1978. Yitrium and lanthanides. [In:] Wedpohl, K. (Ed.), Handbook of Geochemistry. Springer-Verlag, New York, 57-71.

Fourcade, S., Marquer, D. \& Javoy, M., 1989. $18 \mathrm{O} / 16 \mathrm{O}$ variations and fluid circulation in a deep shear zone: the case of the alpine ultramylonites from the Aar massif (Central Alps, Switzerland). Chemical Geology 77, 119-131.

Ghasemi, A. \& Talbot, C.J., 2006. A new tectonic scenario for the Sanandaj-Sirjan Zone (Iran). Journal of Asian Earth Sciences 26, 683-693.

Glazner, A.F. \& Bartley, J.M., 1991. Volume loss fluid flow and state of strain in the extensional mylonites from the central Mojave Desert, California. Journal of Structural Geology 5, 587-594.

Goddard, J.V. \& Evans, J.P., 1995. Chemical changes and fluid-rock interaction in faults of crystalline thrust sheets, northwestern Wyoming, USA. Journal of Structural Geology 17, 533-547.

Goldfarb, R.J., Groves, D.I. \& Gardoll, S., 2001. Orogenic gold and geologic time: a global synthesis. Ore Geology Reviews 18, 1-75.

Goldfarb, R.J., Ayuso, R., Miller, M.L., Ebert, S.W., Marsh, E.E., Petsel, S.A., Miller, L.D., Bradley, D., Johnson, G. \& Mcclelland, W., 2004. The Late Cretaceous Donlin Creek Gold Deposit, Southwestern Alaska: Controls on Epizonal Ore Formation. Economic Geology 99, 643-671.

Goldfarb, R.J., Baker, T., Dube, B., Groves, D.I., Hart, C.J.R. \& Gosselin, P., 2005. Distribution, character and genesis of gold deposits in metamorphic terranes. Economic Geology 100 ${ }^{\text {th }}$ Anniv., 407-450.

Grant, J.A., 1986. The isocon diagram - a simple solution to Gresens' equation for metasomatic alteration. Economic Geology 81, 1976-1982.

Grant, J.A., 2005. Isocon analysis: A brief review of the method and applications. Physics and Chemistry of the Earth 30, 997-1004.

Gresens, R.L., 1967. Composition-volume relationships of metasomatism. Chemical Geology 2, 47-65.

Groves, D.I., Goldfarb, R.J., Gebre, M.M., Hagemann, S.G. \& Robert, F., 1998. Orogenic gold 
deposits: a proposed classification in the context of their crustal distribution and relationship to other gold deposit types. Ore Geology Reviews 13, 7-27.

Groves, D.I., Goldfarb, R.J., Knox-Robinson, C.M., Ojala, J., Gardoll, S., Yun, G. \& Holyland, P., 2000. Late-kinematic timing of orogenic gold deposits and significance for computer-based exploration techniques with emphasis on the Yilgarn block, Western Australia. Ore Geology Reviews 17, 1-38.

Guilbert, J.M. \& Park, C.F., 1997. The geology of ore deposits. W. H. Freeman and Company, New York, 985pp.

Haas, J.R., Shock, E.L. \& Sassani, D.C., 1995. Rare earth elements in hydrothermal systems: estimates of standard partial molal thermodynamic properties of aqueous complexes of the rare earth elements at high pressures and temperatures. Geochimica et Cosmochimica Acta 59, 43294350.

Hagemann, S.G., Gebre-Mariam, G. \& Groves, D.L., 1994. Surface-water influx in shallow-level Archean lode gold deposits in Western Australia. Geology 22, 1067-1070.

Hippertt, J.F., 1998. Breakdown of feldspar, volume gain and lateral mass transfer during mylonitization of granitoid in a low metamorphic grade shear zone. Journal of Structural Geology 20, 175193.

Ingles, J., Lamouroux, C., Soula, J.C., Guerrero, N. \& Debat, P., 1999. Nucleation of ductile shear zone in a granodiorite under greenschist facies conditions, Néouvielle massif, Pyrenees, France. Journal of Structural Geology 21, 555-576.

Irvine, T.N. \& Baragar, W.R.A., 1971. A guide to the chemical classification of the common volcanic rocks. Canadian Journal of Earth Sciences 8, 523-548.

Kerrich, R., Allison, I., Barnett, R.L., Moss, S. \& Starkey, J., 1980. Microstructural and chemical transformations accompanying deformation of granite in a shear zone at Mieville, Switzerland; with implications for stress corrosion cracking and superplastic flow. Contributions to Mineralogy and Petrology 73, 221-241.

Kesler, S.E., 2005. Ore-forming fluids. Elements 1, 13-18.

Kikawada, Y., 2001. Experimental studies on the mobility of lanthanides accompanying alteration of andesite by acidic hot spring water. Chemical Geology 176, 137-149.

Kwon, S., Park, Y., Park, C. \& Kim, H.S., 2009. Mass-balance analysis of bulk-rock chemical changes during mylonitization of a meg- acryst-bearing granitoid, Cheongsan shear zone, Korea. Journal of Asian Earth Sciences 35, 489-501.

Lewis, A.J., Palmer, M.R., Sturchio, N.C. \& Kemp, A.J., 1997. The rare earth element geochemistry of acid-sulphate and acid-sulphate-chloride geothermal systems from Yellowstone National Park, Wyoming, USA. Geochimica et Cosmochimica Acta 61, 695-706.

Lottermoser, B.G., 1992. Rare earth elements and hydrothermal ore formation processes. Ore Geology Reviews 7, 25-41.

McCuaig, T.C. \& Kerrich. R., 1998. P-T-t-deformation-fluid characteristics of lode gold deposits: evidence from alteration systematics. Ore Geology Reviews 12, 381-453.

Michard, A., 1989. Rare earth element systematic in hydrothermal fluid. Geochimica et Cosmochimica Acta 53, 745-750.

Mohajjel, M., 1997. Structure and tectonic evolution of Palaeozoic-Mesozoic rocks, Sanandaj-Sirjan Zone, Western Iran. University of Wollongong, Wollongong, $284 \mathrm{pp}$.

Mohajjel, M., 2000. Geological map of the Qolqoleh and Kervian gold deposits, 1:5000. Geological Survey of Iran.

Mohajjel, M., Fergusson, C.L. \& Sahandi, M.R., 2003. Cretaceous-Tertiary convergence and continental collision, Sanandaj-Sirjan Zone, western Iran. Journal of Asian Earth Sciences 21, 397-412.

Moinevaziri, H., 1985. Volcanisme Tértiaire et Quatérnaire en Iran. [Tertiary and Quaternary volcanism in Iran]. University Paris-Sud, Paris, 290 pp. (in French)

Murray, R.W., Brink, M.R., Jones, D.L., Gerlach, D.C. \& Russ, G.P., 1990. REE as indicators of deffrent marine depositional environments in chert and shale. Geology 18, 268-271.

Nakamura, N., 1974. Determination of REE, Ba, Fe, $\mathrm{Mg}, \mathrm{Na}$ and $\mathrm{K}$ in carbonaceous and ordinary chondrites. Geochimical Acta 38, 757-775.

Nesbitt, B.E., Muehlenbachs, K. \& Murrowchick, J.B., 1989. Genetic implications of the stable isotope characteristics of mesothermal Au deposits and related $\mathrm{Sb}$ and $\mathrm{Hg}$ deposits in the Canadian Cordillera. Economic Geology 84, 1489-1506.

Newman, J. \& Mitra, G., 1993. Lateral variations in mylonite zone thickness as influenced by fluidrock interactions, Linville Falls fault, North Carolina. Journal of Structural Geology 15, 849-863.

Niroomand, S., Goldfarb, R.J., Moore, F., Mohajjel, M. \& Marsh, E.E., 2011. The Kharapeh orogenic gold deposit: geological, structural, and geochemical controls on epizonal ore formation in West Azerbaijan Province, Northwestern Iran. Mineralium Deposita 46, 409-428. 
O’Hara, K., 1988. Fluid flow and volume loss during mylonitization: an origin for phyllonites in an overthrust setting, North Carolina. USA. Tectonophysics 156, 21-36.

O’Hara, K. \& Blackburn, W.H., 1989. Volume-loss model for trace-element enrichments in mylonites. Geology 17, 524-527.

O'Hara, K., 1990. State of strain in mylonites from the western Blue Ridge province, southern Appalachians: the role of volume loss. Journal of Structural Geology 12, 419-430.

Omrani, J., Agard, P., Whitechurch, H., Mathieu, B., Prouteau, G. \& Jolivet, L., 2008. Arc-magmatism and subduction history beneath the Zagros Mountains, Iran. A new report of adakites and geodynamic consequences. Lithos 106, 380-398.

Pattrick, R.A.D., Boyce, A. \& MacIntyre, R.M., 1988. Gold and silver mineralization at Tyndrum, Scotland. Mineralogy and Petrology 38, 61-76.

Pearce, J.A., Harris, N.W. \& Tindle, A.G., 1984. Trace element discrimination diagrams for the tectonic interpretation of granitic rocks. Journal of Petrology 25, 956-983.

Pirajno, F., 2009. Hydrothermal mineral deposits, principle and fundamental concept for the exploration geologist. Springer, 706 pp.

Roedder, E., 1984. Fluid inclusions. Reviews in Mineralogy 12, 644.

Rolland, Y., Cox, S., Boullier, A.M., Pennacchioni, G. \& Mancktelow, N., 2003. Rare earth and tracen element mobility in mid-crustal shear zones: insights from the Mont Blanc Massif (Western Alps). Earth and Planetary Science Letters 214, 203-219.

Rollinson, H.R., 1993. Using geochemical data: evolution, presentation, interpretation. Longman, London, 652 pp.

Sarkarinejad, K. \& Alizadeh, A., 2009. Dynamic model for the exhumation of the Tutak gneiss dome within a bivergent wedge in the Zagros Thrust System of Iran. Journal of Geodynamics 47, 201-209.

Selverstone, J., Morteani, G. \& Stuade, J.M., 1991. Fluid channelling during ductile shearing: transformation of granodiorite into aluminous schist in the Tauern Window, eastern Alps. Journal of Metamorphic Geology 9, 419-431.

Shahabpour, J., 2005. Tectonic evolution of the orogenic belt in the region located between Kerman and Neyriz. Journal of Asian Earth Sciences 24, 405-17.

Sheikholeslami, M.R., Pique, A., Mobayen, P., Sabzehei, M., Bellon, H. \& Hashem Emami, M.,
2008. Tectono-metamorphic evolution of the Neyriz metamorphic complex, Quri-Kor-e-Sefid area (Sanandaj- Sirjan Zone, SW Iran). Journal of Asian Earth Sciences 31, 504-521.

Shepherd, T.J., Rankin, A.H. \& Alderton, D.H.M., 1985. A Practical Guide to Fluid Inclusion Studies. Blackie, Glasgow, 239 pp.

Sinha, A.K., Hewitt, D.A. \& Rimstidt, J.D., 1986. Fluid interaction and element mobility in the development of ultramylonites. Geology 14, 883-886.

So, C.S. \& Yun, S.T., 1997. Jurassic mesothermal gold mineralization of the Samhwanghak mine, Youngdong area, Republic of Korea: constraints on hydrothermal fluid chemistry. Economic Geology 92, 60-80.

Sterner, S.M. \& Bodnar, R.J., 1984. Synthetic fluid inclusions in natural quartz. I. Compositional types synthesized and applications to experimental geochemistry. Geochimica et Cosmochimica Acta 48, 2659-2668.

Taylor, S.R. \& McLennan, S.M., 1985. The Continental Crust: its Composition and Evolution. Blackwell, Oxford, 312 pp.

Touret, J. \& Dietvorst, P., 1983. Fluid inclusions in high-grade anatectic metamorphites. Journal of the Geological Society of London 140, 635-649.

Touret, J.L.R., 2001. Fluids in metamorphic rocks. Lithos 55, 1-26.

Vance, R.K. \& Condie, K.C., 1987. Geochemistry of footwall alteration associated with the early Proterozoic United Veerde massive sulfide deposit, Jerome, Arizona. Economic Geology 82, 571-586.

Wendlandt, R.F. \& Harrison, W.J., 1979. Rare earth partitioning between immiscible carbonate and silicate liquids and $\mathrm{CO} 2$ vapour: results and implications for the formation of light rare-earth-enriched rocks. Contributions to Mineralogy and Petrology 69, 409-419.

Wilkinson, J.J., 2001. Fluid inclusions in hydrothermal ore deposits. Lithos 55,229-272.

Wood, S.A., 1990. The aqueous geochemistry of the rare-earth elements and Yttrium. 2. Theoretical predictions of speciation in hydrothermal solutions to $350^{\circ} \mathrm{C}$ at saturation water vapour pressure. Chemical Geology 88, 99-125.

Zhang, X.H., Liu, Q., Ma, Y.J. \& Wang, H., 2005. Geology, fluid inclusions, isotope geochemistry, and geochronology of the Paishanlou shear zone-hosted Gold Deposit, North China Craton. Ore Geology Reviews 26, 325-348.

Manuscript submitted 3 July 2014 Revision accepted 28 January 2015 